This item was submitted to Loughborough's Research Repository by the author.

Items in Figshare are protected by copyright, with all rights reserved, unless otherwise indicated.

\title{
Modeling the structure of liquids and crystals using one- and two-component modified phase-field crystal models
}

PLEASE CITE THE PUBLISHED VERSION

http://dx.doi.org/10.1103/PhysRevE.85.061408

PUBLISHER

(C) American Physical Society

VERSION

VoR (Version of Record)

LICENCE

CC BY-NC-ND 4.0

\section{REPOSITORY RECORD}

Robbins, Mark J., Andrew J. Archer, Uwe Thiele, and Edgar Knobloch. 2019. "Modeling the Structure of Liquids and Crystals Using One- and Two-component Modified Phase-field Crystal Models". figshare. https://hdl.handle.net/2134/12310. 
This item was submitted to Loughborough's Institutional Repository (https://dspace.lboro.ac.uk/) by the author and is made available under the following Creative Commons Licence conditions.

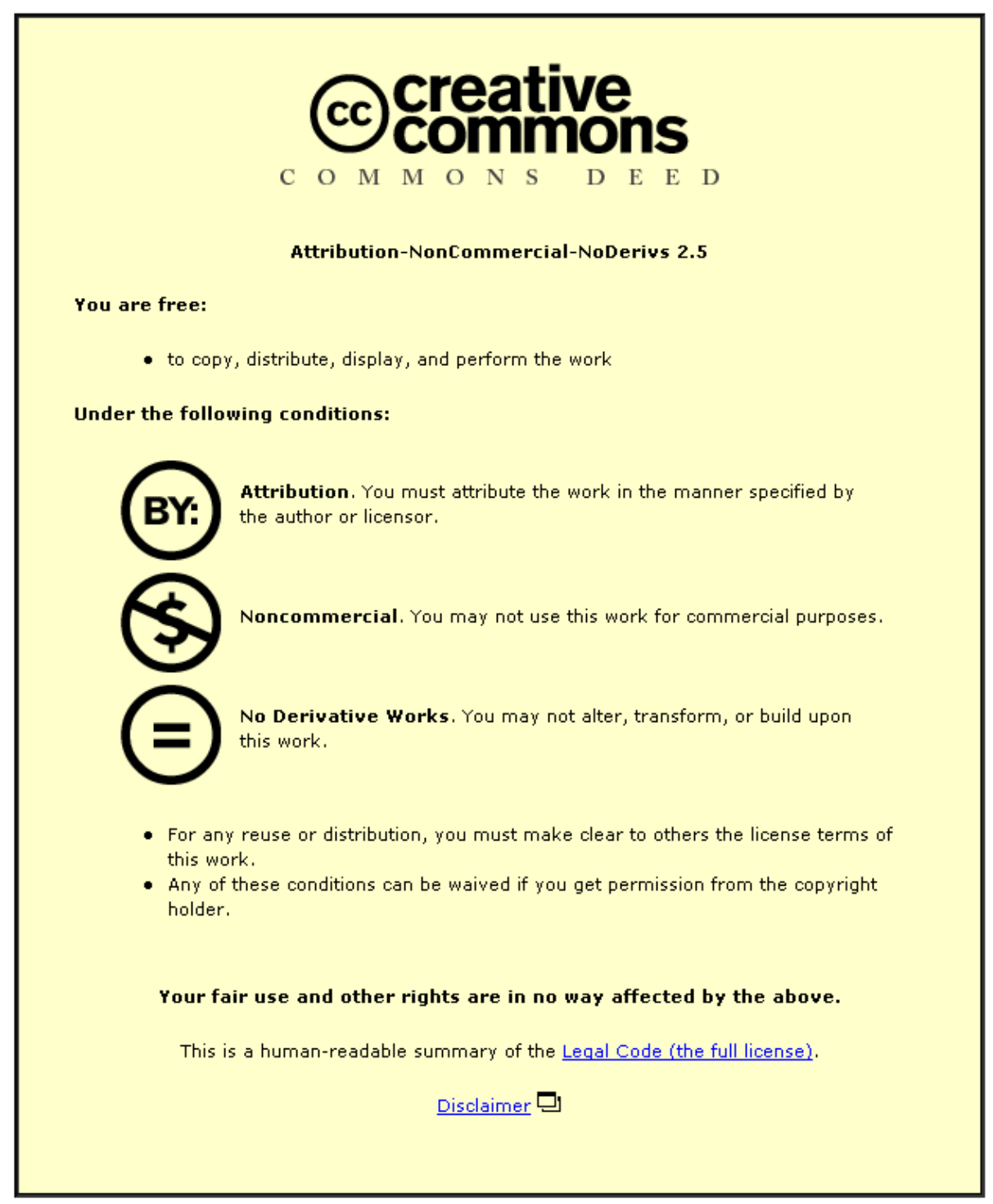

For the full text of this licence, please go to: http://creativecommons.org/licenses/by-nc-nd/2.5/ 


\title{
Modeling the structure of liquids and crystals using one- and two-component modified phase-field crystal models
}

\author{
M. J. Robbins, ${ }^{1}$ A. J. Archer, ${ }^{1}$ U. Thiele,${ }^{1}$ and E. Knobloch ${ }^{2}$ \\ ${ }^{1}$ Department of Mathematical Sciences, Loughborough University, Leicestershire LE11 3TU, United Kingdom \\ ${ }^{2}$ Department of Physics, University of California, Berkeley, California 94720, USA
}

(Received 28 March 2012; published 21 June 2012)

\begin{abstract}
A modified phase-field crystal model in which the free energy may be minimized by an order parameter profile having isolated bumps is investigated. The phase diagram is calculated in one and two dimensions and we locate the regions where modulated and uniform phases are formed and also regions where localized states are formed. We investigate the effectiveness of the phase-field crystal model for describing fluids and crystals with defects. We further consider a two-component model and elucidate how the structure transforms from hexagonal crystalline ordering to square ordering as the concentration changes. Our conclusion contains a discussion of possible interpretations of the order parameter field.
\end{abstract}

DOI: 10.1103/PhysRevE.85.061408

PACS number(s): 82.70.Dd, 61.50.Ah, 05.70.Fh, 02.30.Oz

\section{INTRODUCTION}

Modeling materials at the atomic scale is a task which, for example, may be performed using molecular dynamics simulations. This involves solving coupled equations of motion to calculate the position of each particle at every time step. The resulting calculations can be very computationally expensive, especially when one seeks to consider phenomena which involve a large number of particles. Only short atomic time scales can be feasibly accessed with this or other such approaches. However, there are some instances where it is important to consider materials on the atomic length scale for much longer diffusive time scales, for example, when investigating freezing or glass transitions. One approach to such problems that may be adopted is to develop a phase-field model capable of describing the structure of materials on the scale of the individual particles. In contrast to traditional phase-field models, the recently developed phase-field crystal (PFC) models are capable of just such a description and are now widely used in the literature to model crystalline structures [1-5]. The PFC model consists of a Swift-Hohenberglike equation [6], but with conserved dynamics rather than the nonconserved dynamics of the regular Swift-Hohenberg equation. Similar models with conserved dynamics arise in quite different contexts as well [7,8]. The regular PFC model is governed by the equation

$$
\frac{\partial \phi(\mathbf{x}, t)}{\partial t}=\alpha \nabla^{2} \frac{\delta F[\phi]}{\delta \phi(\mathbf{x}, t)},
$$

where the free energy functional

$$
F[\phi]=\int d \mathbf{x} f(\phi),
$$

with

$$
f(\phi)=\frac{\phi}{2}\left[r+\left(q^{2}+\nabla^{2}\right)^{2}\right] \phi+\frac{\phi^{4}}{4},
$$

where $\alpha$ is the mobility coefficient, $r$ is the undercooling parameter that decreases with decreasing temperature, $q$ is a constant which determines the typical microscopic length scale in the system, and $\phi(\mathbf{x}, t)$ is the order parameter. For certain parameter values this free energy functional is minimized by an order parameter profile consisting of a periodic array of bumps which somewhat resembles the density distribution of particles in a crystalline material. This interpretation is bolstered by the fact that it has been shown that the PFC model [Eqs. (1) and (2)] may be derived from the density functional theory of freezing [9] and the dynamical density functional theory for colloidal particles $[10,11]$ with certain approximations. The free energy is minimized by either periodic structures or by a homogeneous flat profile, depending on the values of $q, r$, and $\bar{\phi}=\frac{1}{L^{d}} \int d \mathbf{x} \phi(\mathbf{x}, t)$, where $L^{d}$ is the size of the system. In two dimensions $(d=2)$, four different phases are observed: a homogeneous phase, two hexagonal phases (one consisting of hexagonally ordered bumps and the other of hexagonally ordered holes), and a stripe phase $[1-3,12]$. The literature largely focuses on the region of the two-dimensional (2D) phase diagram which contains the hexagonally arranged bumps and their transition to the homogeneous state $[1-5,9,10,12]$. The uniform profile $\phi(\mathbf{x}, t)=\bar{\phi}$ represents the order parameter in a uniform liquid and the hexagonal phase is treated as a crystal. The model is then used to consider a number of problems including melting and freezing $[3,9,10]$ and grain boundary effects $[1,2,4]$.

In this "standard" PFC model [Eqs. (1)-(3)] the hexagonally arranged bumps are considered to be particles or colloids in a crystalline structure. In this context, the physical interpretation of the striped and hexagonally ordered hole structures is unclear and, as such, these phases are commonly ignored. The conjecture that the ordered bumps represent crystalline particle structures can be extended by including a "vacancy term" in the free energy $[13,14]$, which strongly breaks the hole-bump ( $\phi \rightarrow-\phi)$ symmetry of Eq. (3):

$$
F[\phi]=\int d \mathbf{x}\left[f(\phi)+f_{\mathrm{vac}}(\phi)\right] .
$$

Using the free energy (4), it is possible to obtain structures which contain a mixture of bumps and vacant areas (the "vacancies" are areas where the order parameter is approximately uniform around the value $\phi \approx 0$ ). As the system parameters are varied, one can observe states where the number of bumps changes and the proportion of the system in which $\phi \approx 0$ varies. In particular, one can go from states where the density 
of bumps is high, vacancies are absent, and the bumps are hexagonally ordered, much as in the standard PFC model. However, for other parameter values, the density of bumps is significantly lower, with more vacant $\phi \approx 0$ regions and no long range order in the location of the bumps [13]. The arrangement of the bumps in these states resembles snapshots of the particles in a fluid. The interpretation that the bumps represent particles and that this "vacancy phase-field crystal" (VPFC) may be used to model particle configurations on a coarse-grained scale of both crystalline solids and disordered fluids is the central assumption of Ref. [13]. We return to the issue of the precise interpretation of the nature of the order parameter field in the conclusion.

In this paper we investigate the thermodynamics and the structures formed in the augmented conserved SwiftHohenberg model with the free energy functional (4), hereafter the VPFC model, and extend the theory to a two-component generalization of this model. The vacancy term takes the following form $[13,14]$ :

$$
f_{\mathrm{vac}}(\phi)=H \phi^{2}(|\phi|-\phi),
$$

where $H$ is a constant. We use the value $H=1500$, as in Refs. [13,14], although the precise value of $H$ is not significant. We have confirmed that choosing $H$ in the range 500-3000 makes no qualitative difference to the structures formed. This vacancy term acts as a piecewise function which is zero for positive values of $\phi$ and takes an increasingly large value when $\phi<0$. Hence, this term penalizes negative values of $\phi$. This leads to the VPFC model forming periodic structures which are somewhat different from those of the regular PFC. In addition, the VPFC model has a large region of parameter space at small $\bar{\phi}$, where spatially localized structures form. The time evolution of the order parameter $\phi$ is governed by the conserved dynamics used in the standard PFC model (1).

We begin in Sec. II by considering the phase behavior of the model, investigating the transition between periodic and localized states. We focus on understanding the bifurcation diagrams connecting the various uniform, periodic, and localized states exhibited by the model. We then go on to consider how individual localized states or particles interact with one another. In Sec. III we extend the model to consider a two-component system, and we determine how the particles in the binary mixture interact with one another. We find a transition between hexagonal and square ordering of the particles as the concentration changes. Our conclusions follow in Sec. IV and include a discussion of the proper interpretation of the order parameter field $\phi$.

\section{ONE-COMPONENT SYSTEM}

\section{A. Linear stability of a homogeneous profile}

We begin by considering the phase behavior of the VPFC model [Eqs. (1) and (4)]. We calculate the limit of linear stability for a homogeneous flat state using a linear stability analysis. In the context of colloidal suspensions exhibiting microphase separation and fluids of charged particles, this limit of linear stability is referred to as a " $\lambda$ line" [15-18]. Since $f_{\text {vac }}$ is nondifferentiable at $\phi=0$ we treat it in a piecewise manner, by treating the two cases $\bar{\phi}>0$ and $\bar{\phi}<0$ separately [in fact, if $\phi(\mathbf{x})$ takes the form of Eq. (6) and $\bar{\phi}>|\xi|$, then $f_{\text {vac }}=0$ everywhere and the thermodynamics of the VPFC model reduces to that of the regular PFC model]. We assume that the order parameter $\phi$ takes the form of a flat profile plus an additional small-amplitude harmonic modulation:

$$
\phi=\bar{\phi}+\delta \phi=\bar{\phi}+\xi e^{i \mathbf{k} \cdot \mathbf{x}} e^{\beta t},
$$

where $\bar{\phi}$ is the average value of the order parameter and the amplitude $|\xi| \ll 1$. Substituting this expression into the functional derivative of the free energy (4) we obtain

$$
\begin{aligned}
\frac{\delta F}{\delta \phi}= & \left(r+q^{4}\right) \bar{\phi}+3 H \bar{\phi}(|\bar{\phi}|-\bar{\phi})+\bar{\phi}^{3} \\
& +\left[\left(k^{2}-q^{2}\right)^{2}+\Delta\right] \delta \phi+O\left(\delta \phi^{2}\right),
\end{aligned}
$$

where

$$
\Delta=r+6 H(|\bar{\phi}|-\bar{\phi})+3 \bar{\phi}^{2} .
$$

Inserting this expression for the functional derivative (7) into the dynamical equation (1) and then linearizing we arrive at the following dispersion relation:

$$
\beta=-k^{2} \alpha\left[\left(k^{2}-q^{2}\right)^{2}+\Delta\right] .
$$

When the growth rate $\beta(k)>0$, any small amplitude modulation with wave number $k=|\mathbf{k}|$ will grow over time. There is a local maximum in $\beta$ (which becomes the global maximum when the uniform state is unstable) at the wave number:

$$
k_{m}=\frac{1}{3} \sqrt{6 q^{2}+3 \sqrt{q^{4}-3 \Delta}} .
$$

Thus, if one takes an initially almost flat profile $\phi(\mathbf{x}, t=$ $0)=\bar{\phi}+\mathcal{X}(\mathbf{x})$, where $\mathcal{X}(\mathbf{x})$ is composed of a sum of a large number of small-amplitude harmonic modulations [cf. Eq. (6)] with different wave numbers $k$ [in practice $\mathcal{X}(\mathbf{x})$ is generated by adding a small random number to the discretized initial profile], then as the system evolves in time $\phi(\mathbf{x}, t)$ will develop spatial modulation on the length scale $\frac{2 \pi}{k_{m}}$, since this scale corresponds to the maximum growth rate $\beta_{m} \equiv \beta\left(k_{m}\right)$. This length scale has an inverse dependence on the value of $q$; that is, increasing the value of $q$ reduces the length scale of the structures which are formed.

The limit of linear stability is defined as the locus of points in parameter space where the maximum in the dispersion relation (9) is at zero, that is, $\beta_{m}=0$. The conditions $\beta=$ $\frac{\partial \beta}{\partial k}=0$, subject to the requirement that $k_{m} \neq 0$ yield $\Delta=0$, $k_{m}= \pm q$. Thus, $\Delta$ in Eq. (8) can be considered as a measure of stability: When $\Delta<0$ the system is linearly unstable and when $\Delta>0$ the system is linearly stable. The magnitude of $\Delta$ indicates how "far" we are from the limit of stability. Figure 1 shows the dispersion relations $\beta(k)$ for various values of $\Delta$. In accordance with Eq. (10), the maximum at $k_{m} \approx q$ disappears when $\Delta>\frac{q^{4}}{3}$; in this case only the maximum at $k=0$ remains. It is important to note that these results are identical to those of the regular PFC model [Eqs. (1) and (2)] for positive values of the order parameter $\bar{\phi}>0$.

\section{B. One-dimensional model}

In order to develop a better understanding of the effect of the "vacancy term" (5) we initially consider the phase diagram for the system in one spatial dimension. The regular PFC model [Eqs. (1) and (2)] in 1D exhibits two distinct 


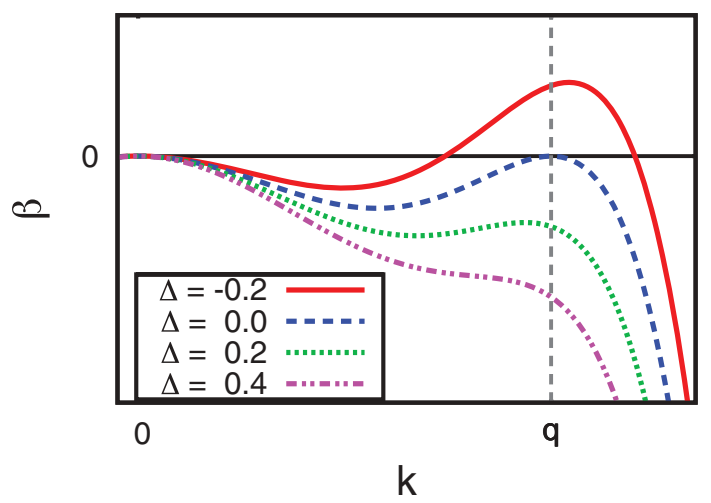

FIG. 1. (Color online) Dispersion relation curves for the VPFC model [Eqs. (1) and (4)] when $q=1$. Four cases are shown, with (i) $\beta\left(k_{m}\right)>0$ (red solid line), (ii) $\beta\left(k_{m}\right)=0$ (blue dashed line), (iii) $\beta\left(k_{m}\right)<0$ but $k_{m}>0$ (green dotted line), and (iv) $\beta \leqslant 0$ and $k_{m}=0$ (magenta dash-dotted line).

phases [2]: a nonuniform state in which the order parameter profile resembles a sinusoid and a uniform state in which the order parameter is a constant. The phase diagram of the regular PFC model is symmetric around $\bar{\phi}=0$ owing to the symmetry of the free energy (2) with respect to $\phi \rightarrow-\phi$. This is no longer the case when the vacancy term (5) is added.

The phase diagram for the 1D VPFC model is shown in Fig. 2(a) and is very different from that of the regular PFC [2]. As with the regular PFC model, modulated profiles are present below the limit of linear stability $\Delta=0$ (blue dashed line) provided $\bar{\phi}<\sqrt{3 / 2} q^{2}$. However, with the added vacancy term (5) the lower limit for the presence of the modulated phase is at $\bar{\phi} \gtrsim 0(H \gg 1)$. The tricritical point with $\bar{\phi}>0$ (red dot) familiar from the PFC model remains. Above this point the phase transition between the periodic and homogeneous phases is of second order. Below this point a periodic phase with $\bar{\phi}=\bar{\phi}_{p}$ coexists with a homogeneous phase with $\bar{\phi}=\bar{\phi}_{h}$ and the phase transition between these phases is of first order. Figure 2(a) shows the coexisting phases using fixed temperature (horizontal) tie lines connecting $\bar{\phi}_{p}$ and $\bar{\phi}_{h}$ (solid red lines).

We can calculate the location of the tricritical point as follows: Since the wave number near $\Delta=0$ is $k_{m} \approx q$ we assume that the order parameter profile takes the form

$$
\phi=\bar{\phi}_{p}+A \cos q x+B \cos 2 q x+\cdots
$$

and compute the free energy $F$. When $\bar{\phi}_{p}>0$ the vacancy term drops out and we obtain the following expression for the free energy per unit length $f_{p}=F / L$ of the periodically modulated phase:

$$
\begin{aligned}
f_{p}= & \frac{1}{2}\left(r+q^{4}\right) \bar{\phi}_{p}^{2}+\frac{1}{4} \bar{\phi}_{p}^{4}+\frac{1}{4} r A^{2}+\frac{1}{4}\left(r+9 q^{4}\right) B^{2} \\
& +\frac{3}{4} \bar{\phi}_{p}^{2}\left(A^{2}+B^{2}\right)+\frac{3}{4} \bar{\phi}_{p} A^{2} B+\frac{3}{32}\left(A^{4}+4 A^{2} B^{2}+B^{4}\right) .
\end{aligned}
$$

We refer to the ansatz (11) as the two-mode approximation. This approximation is reliable around and above the tricritical point since the amplitude of the modulation in $\phi$ is small when $|\Delta| \ll 1$. Moreover the two-mode approximation appears to be exact at the tricritical point, since the location of the tricritical point is unaffected by the inclusion of $\cos 3 q x$ and other higher order modes. In contrast, the mode $B \cos 2 q x$ must be retained in order to obtain the correct value of the amplitude $A$ in the vicinity of the tricritical point (see below).

To demonstrate this we minimize $f_{p}$ in Eq. (12) with respect to the amplitudes $A$ and $B$, obtaining the following two conditions:

$$
\begin{gathered}
r+3 \bar{\phi}_{p}^{2}+3 \bar{\phi}_{p} B+\frac{3}{4} A^{2}+\frac{3}{2} B^{2}=0, \\
\left(r+9 q^{4}\right) B+3 \bar{\phi}_{p}^{2} B+\frac{3}{2} \bar{\phi}_{p} A^{2}+\frac{3}{2} A^{2} B+\frac{3}{4} B^{3}=0 .
\end{gathered}
$$

Solving these for the amplitudes $A$ and $B$ and substituting into Eq. (12), we obtain an approximation for the free energy density of the periodic phase $f_{p}$. Linearizing Eq. (14) in $B$, we find that

$$
B=-\frac{\bar{\phi}_{p} A^{2}}{6 q^{4}}+O\left(\Delta_{p} A^{2}, A^{4}\right),
$$

where $\Delta_{p} \equiv r+3 \bar{\phi}_{p}^{2}$ and hence, from Eq. (13), that

$$
A=2 \sqrt{-\frac{\Delta_{p}}{3}}\left(1-\frac{2 \bar{\phi}_{p}^{2}}{3 q^{4}}\right)^{-1 / 2}+O\left(\Delta_{p}\right) .
$$

The free energy density of the homogeneous phase $f_{h}$, having $\phi(x)=\bar{\phi}_{h}$, is obtained simply setting $A=B=0$ in Eq. (12) to obtain $f_{h}=\frac{1}{2}\left(r+q^{4}\right) \bar{\phi}_{h}^{2}+\frac{1}{4} \bar{\phi}_{h}^{4}$. The chemical potential in the homogeneous phase is $\mu_{h}=\partial f_{h} / \partial \bar{\phi}_{h}$, and in the periodic phase $\mu_{p}=\partial f_{p} / \partial \bar{\phi}_{p}$.

To calculate the location of the tricritical point we recall that at coexistence between the periodic state and the homogeneous state we must have $\mu_{p}=\mu_{h}$. We write the average value of $\phi$ in the periodic state $\bar{\phi}_{p}=\bar{\phi}_{h}+C$, where $C$ is the difference between the average value of the order parameter in the two coexisting phases, implying that the coexistence condition is

$$
\mu_{p}\left(\bar{\phi}_{h}+C\right)-\mu_{h}\left(\bar{\phi}_{h}\right)=0,
$$

or equivalently,

$$
\left(r+q^{4}\right) C+3 \bar{\phi}_{h}^{2} C+\frac{3}{2} \bar{\phi}_{h} A^{2}+O\left(A^{4}, C A^{2}, C^{2}\right)=0 .
$$

Since the amplitude $A$ of the modulated phase at coexistence is small when $\left|\Delta_{h}\right| \ll 1$, where $\Delta_{h} \equiv r+3 \bar{\phi}_{h}^{2}$, Eqs. (14) and (18) yield, for $\left|\Delta_{h}\right| \ll 1$, the expressions

$$
\begin{gathered}
B=-\frac{\bar{\phi}_{h} A^{2}}{6 q^{4}}+O\left(\Delta_{h} A^{2}, A^{4}\right), \\
C=-\frac{3 \bar{\phi}_{h} A^{2}}{2 q^{4}}+O\left(\Delta_{h} A^{2}, A^{4}\right) .
\end{gathered}
$$

Equation (13) then yields

$$
A=2 \sqrt{-\frac{\Delta_{h}}{3}}\left(1-\frac{38 \bar{\phi}_{h}^{2}}{3 q^{4}}\right)^{-1 / 2}+O\left(\Delta_{h}\right) .
$$

Taking the limit $C \rightarrow 0$ now takes us to the tricritical point. At the tricritical point $\Delta_{h}=\Delta_{p}=0$ and the chemical potentials $\mu_{p}\left(\bar{\phi}_{h}\right)$ and $\mu_{h}\left(\bar{\phi}_{h}\right)$ are identical. Thus, from Eq. (20) we see that the tricritical point occurs at $\bar{\phi}=\sqrt{3 / 38} q^{2} \approx 0.281 q^{2}$, $r=-(9 / 38) q^{4} \approx-0.237 q^{4}$. These coordinates agree precisely with the result obtained from the common tangent construction between the free energy of the periodic phase and the free energy of the homogeneous phase to determine phase coexistence and also with our numerical simulations of 


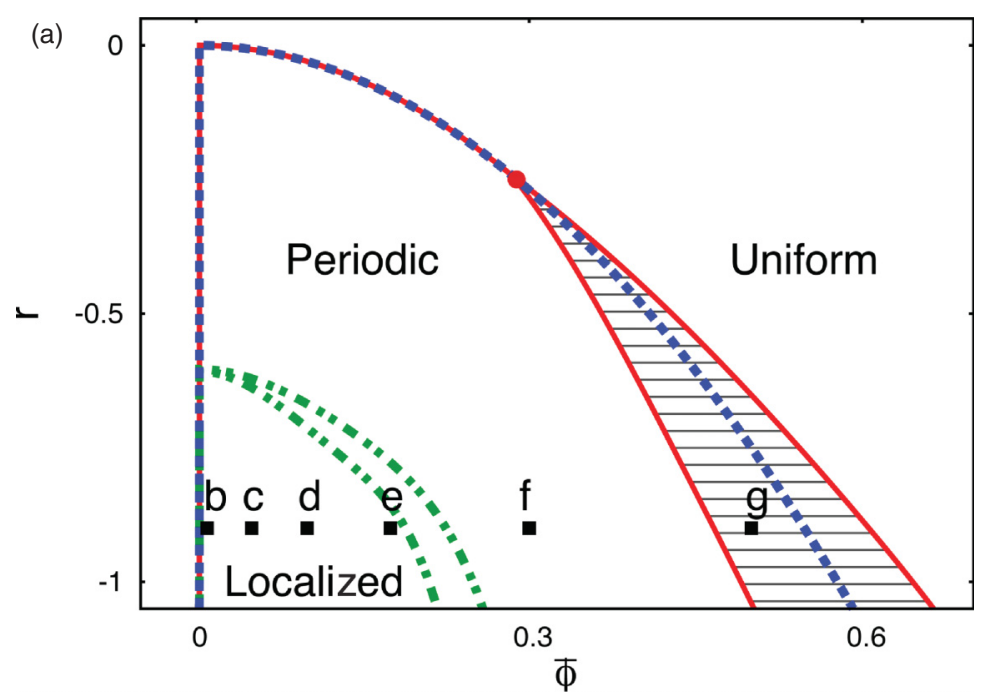

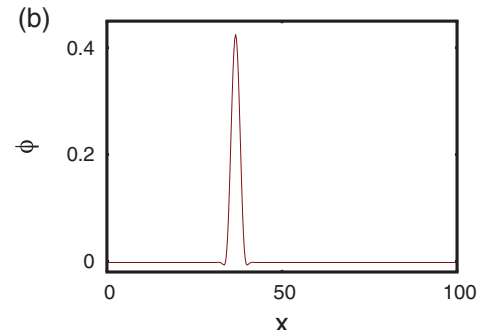

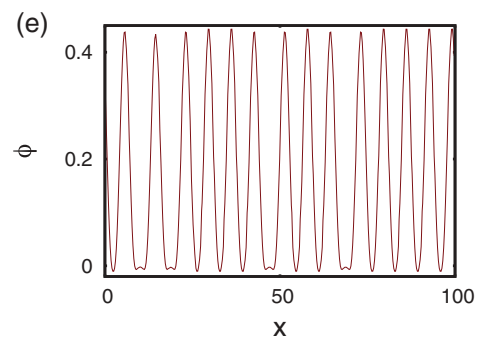

(c)

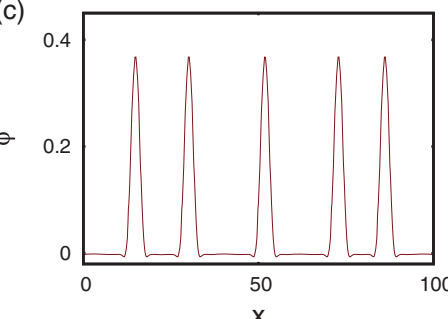

(f)

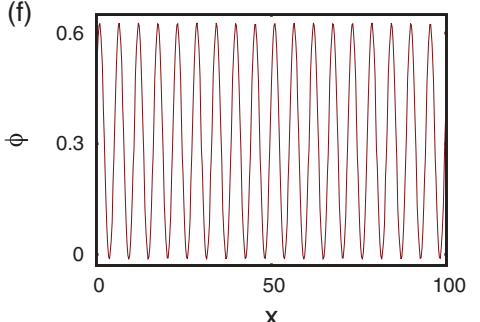

(d)

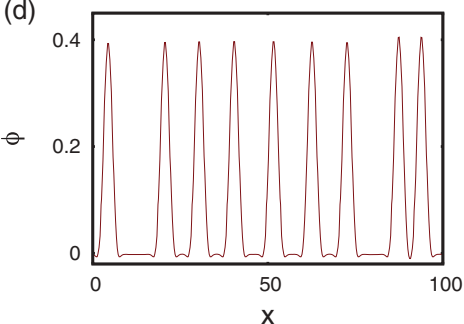

(g)

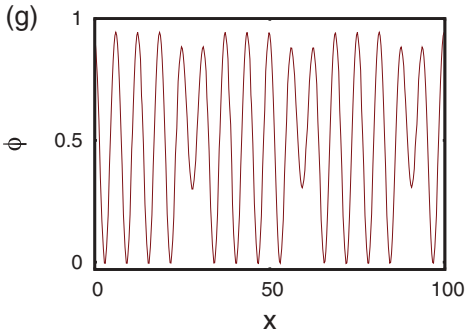

FIG. 2. (Color online) The phase diagram for the 1D VPFC model [Eqs. (1) and (4)] is displayed in (a) for the case $q=1$. The red solid lines are the coexistence curves between the periodic and uniform phases; the red circle is the tricritical point. The blue dashed line is the locus $\Delta=0$, which is the limit of linear stability for uniform profiles. The green dot-dashed lines are a guide showing the parameter space where local and periodic structures are formed. (b)-(g) Examples of order parameter profiles from numerical simulations corresponding to (local) minima of the free energy for the values of $\bar{\phi}$ and $r$ indicated in (a). The parameter values are $q=1, r=-0.9, \alpha=1$ and (b) $\bar{\phi}=0.01$, (c) $\bar{\phi}=0.05$, (d) $\bar{\phi}=0.1$, (e) $\bar{\phi}=0.175$, (f) $\bar{\phi}=0.3$, and (g) $\bar{\phi}=0.5$.

the VPFC model performed with $q=1$. Thus, when $q=1$ the phase transition is of second order for $r>-9 / 38$ and of first order for $r<-9 / 38$. As already mentioned, this result is exact in the sense that it is unchanged if more modes are included in the ansatz (11) and improves on the prediction $r=-1 / 4$ obtained for the PFC model using a one-mode approximation [2]. Note that the vacancy term (5) does not affect the transition because $\phi$ is positive everywhere.

As discussed above, the transition between the periodic and uniform phases is of first order below the tricritical point. As $r$ decreases, this region of the phase diagram is increasingly affected by the vacancy term (5), as the amplitude of the structures becomes large enough to reach negative $\phi$ values. We observe that including the vacancy term (5) decreases the distance between the coexistence curves. This is because the vacancy term increases the free energy of the profiles in the periodic phase, which decreases the difference between the free energy of the periodic structures and the homogeneous state and hence a common tangent construction between the two yields values which are closer to the linear stability line $\Delta=0$. We calculate the coexistence values below the tricritical point by numerically solving for the order parameter profiles because the two-mode approximation becomes inaccurate when $r<-0.3$, where the order parameter profile develops regions where $\phi<0$ and so the vacancy term makes a contribution to the free energy. Note that for the regular PFC model (i.e., when $H=0$ ) the two-mode approximation for the free energy works very well, agreeing to two significant figures or more with the exact free energy for $r \geqslant-0.9$.

To calculate the coexisting phases numerically we select a value of $r$ and determine the order parameter profile along this line for different values of $\bar{\phi}$. The free energy at each of these points is minimized with respect to the domain size, which effectively gives us the minimum free energy for the infinite 
system. A polynomial is then fitted to these values to produce a continuous curve which gives the free energy of the periodic phase for the chosen value of $r$. We then make the common tangent construction between the free energy of the periodic phase and the uniform phase to calculate the two coexisting $\bar{\phi}$ values at the chosen value of $r$. This process is then repeated for different values of $r$. The resulting coexistence curves are plotted as the solid red lines in Fig. 2(a).

The periodic structures which are formed by the VPFC model [Fig. 2(f)] are qualitatively very similar to the structures which can be found in the regular PFC model. However, the amplitude of the modulations is restricted by the large penalty in the free energy accumulated when $\phi<0$. Inside the coexistence region between the periodic and uniform states, we observe interesting structures where the amplitude of the peaks does not remain constant and a second length scale is visible in the structures [Fig. 2(g)]. This is also an effect which is present in the regular PFC model and will be discussed in detail in future work. What is most intriguing, and is perhaps the most appealing aspect of the VPFC model, is the appearance of localized states for small positive values of $\bar{\phi}$ when the magnitude of $r$ is sufficiently large $(r \lesssim-0.6)$. We obtain order parameter profiles by numerically integrating forward in time Eqs. (1) and (4) until a stationary solution is reached, starting from the initial profile $\phi(\mathbf{x}, t=0)=\bar{\phi}+\mathcal{X}(\mathbf{x})$, where $\mathcal{X}$ is a small amplitude random noise profile with zero mean. A rich variety of different patterns is observed, including periodic structures mixed with almost flat regions [Fig. 2(d)] and individual isolated peaks [Figs. 2(b) and 2(c)]. In Fig. 2(a) the green dot-dashed curves indicate the boundary of the region where one observes regular periodic structures and where the localized structures are formed. Note that these are guidelines only and are not thermodynamic coexistence curves. The lower-left dot-dashed curve roughly denotes the linear stability limit of the regular periodic structures, such as that in Fig. 2(f). This is determined numerically. We begin with a periodic profile and reduce the value of $\bar{\phi}$ gradually, minimizing the free energy at each step, while keeping $r$ constant. The limit point is then defined as the value of $\bar{\phi}$ where the periodic profile becomes linearly unstable and a vacancy is introduced. In a similar way, we determine the upper-right dot-dashed line, which is the limit of linear stability of the structures with defects. This is found by starting with a profile containing a single vacancy and increasing $\bar{\phi}$ until the vacancy disappears. These two points are calculated for different values of $r$ and then a best fit to this data is shown in Fig. 2(a). There is some hysteresis in the region between these two curves, with the type of profile produced depending heavily upon the initial conditions.

Within the localized state region of the phase diagram it is possible to obtain order parameter profiles with a varying number of peaks for a given system of length $L$. Keeping $r \lesssim$ -0.6 constant and varying $\bar{\phi}$ allows us to control the number (density) of bumps, as shown in Figs. 2(b)-2(e). Beginning with $\bar{\phi}=0$ we find isolated peaks in large vacant areas (where $\phi$ is approximately uniform with $\phi \approx 0$ ). As $\bar{\phi}$ is increased the number of peaks increases until we return to the familiar regular periodic structures. The assumption of Ref. [13] is that, unlike in the regular PFC, where the uniform phase is associated with the liquid and the modulated phase with the crystal, in the VPFC model one may associate each bump in $\phi(\mathbf{x})$ as corresponding to a particle, so the model can describe fluids [Figs. 2(c) and 2(d)], crystals with vacancies and defects [Fig. 2(e)], and regular crystals [Fig. 2(f)]. Note that the precise value of the parameter $H$ in the free energy has no qualitative effect on the structures that are formed. The main effect of varying $H$ is to change slightly the value of $\phi$ in the vacant areas between the bumps. For example, for $\bar{\phi}=0.02$ and $r=$ -0.9 , when $H=500$ we find that $\phi \approx-5.3 \times 10^{-3}$ in the vacant areas, when $H=1500$ (the value used for much of the work presented here) we find $\phi \approx-3.0 \times 10^{-3}$, and when $H=3000$ we find that $\phi \approx-2.1 \times 10^{-3}$ in the vacant areas.

The findings presented in Fig. 2 indicate the existence of a hysteretic transition between periodic and localized states and are a consequence of homoclinic snaking [19-22] in the present system. In the standard homoclinic scenario such localized states are present within a part of the coexistence region called the pinning region. The localized states in the lower left part of the parameter plane $(\bar{\phi}, r)$ in Fig. 2(a) correspond to the global energy minimum or to other deep but local energy minima. Families of such steady state solutions can be obtained for the VPFC model that we study here [Eq. (1) with Eqs. (3), (4), and (5)] by employing the path continuation techniques bundled in the package AUTO07P [23]. As an example, in Figs. 3 and 4 we show the characteristics of localized solutions along cuts through the plane $(\bar{\phi}, r)$. In particular, Figs. 3 and 4 give results for changing $r$ (at constant $\bar{\phi}=0.1$ ) and changing $\bar{\phi}$ (at constant $r=-0.9)$, respectively. All solutions are characterized by their $L^{2}$ norm $\|\delta \phi\| \equiv \sqrt{(1 / L) \int_{0}^{L}(\phi(x)-\bar{\phi})^{2} d x}$, chemical potential $\mu=\delta F / \delta \phi$, mean free energy density difference $\left(F[\phi(x)]-F_{0}\right) / L$, where $F_{0}=F[\bar{\phi}]$, and mean grand potential density $\omega \equiv F[\phi(x)] / L-\bar{\phi} \mu$, and satisfy periodic boundary conditions on the domain $0 \leqslant x \leqslant L$.

It turns out that there exist three types of localized steady states. (i) The heavy solid black line consists of $x \rightarrow-x$ symmetric localized states that have a maximum at the center; that is, the overall number of bumps within the structure is odd. (ii) The dashed red line also represents $x \rightarrow-x$ symmetric localized states but this time with a hole (minimum) at the center. (iii) The localized solutions of the third type are not symmetric under $x \rightarrow-x$ and are called "asymmetric states." These reside on branches that connect (via pitchfork bifurcations) the two branches of symmetric localized states. These branches are included in the bifurcation diagrams as dotted green lines.

Examples of order parameter profiles of types (i)-(iii) are presented in Fig. 5, corresponding to the various solution branches displayed in Fig. 4. This sequence of profiles expands upon the few examples shown in Figs. 2(b)-2(g). Recall, however, that the results in Figs. 2(b)-2(g) are obtained starting from an order parameter profile with a small amplitude random noise and so they do not always exactly agree with the steady states at the same $\bar{\phi}$ resulting from the path continuation. The $L^{2}$ norm, chemical potential $\mu$, mean free energy density $\left(F-F_{0}\right) / L$, and the mean grand potential $F / L-\bar{\phi} \mu$ have been calculated for the profiles obtained from time simulations [Figs. 2(b)-2(f)] and are plotted as blue dots in Fig. 4. A close inspection reveals that the energy of the time simulation results is often slightly higher than that from the continuation results, 
(a)
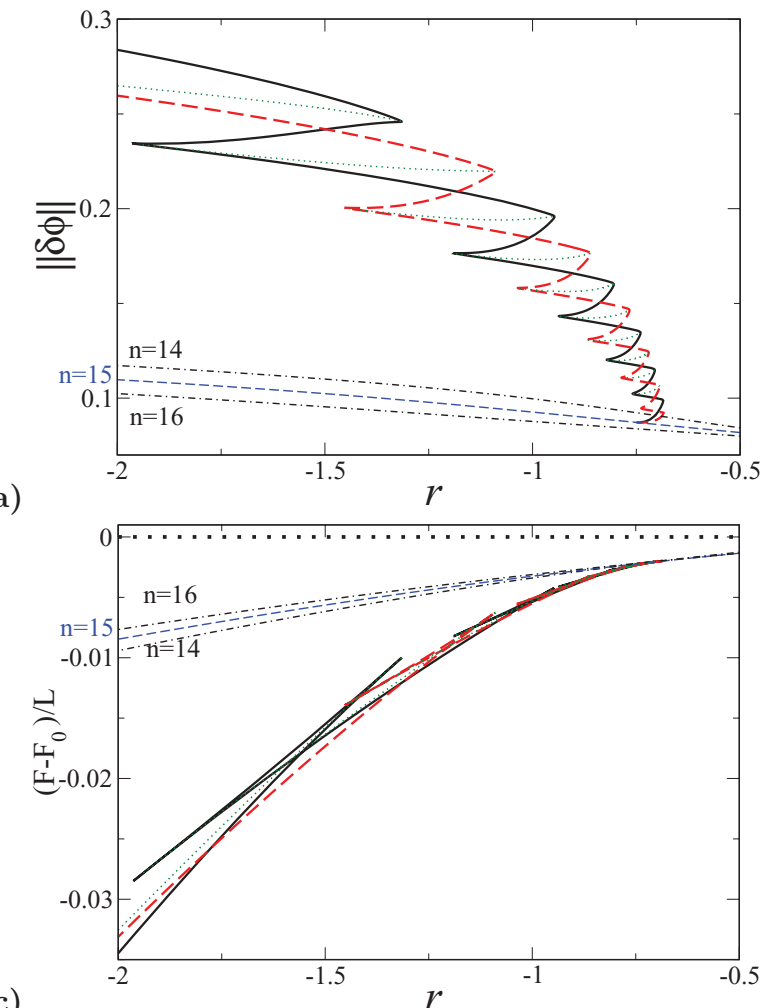
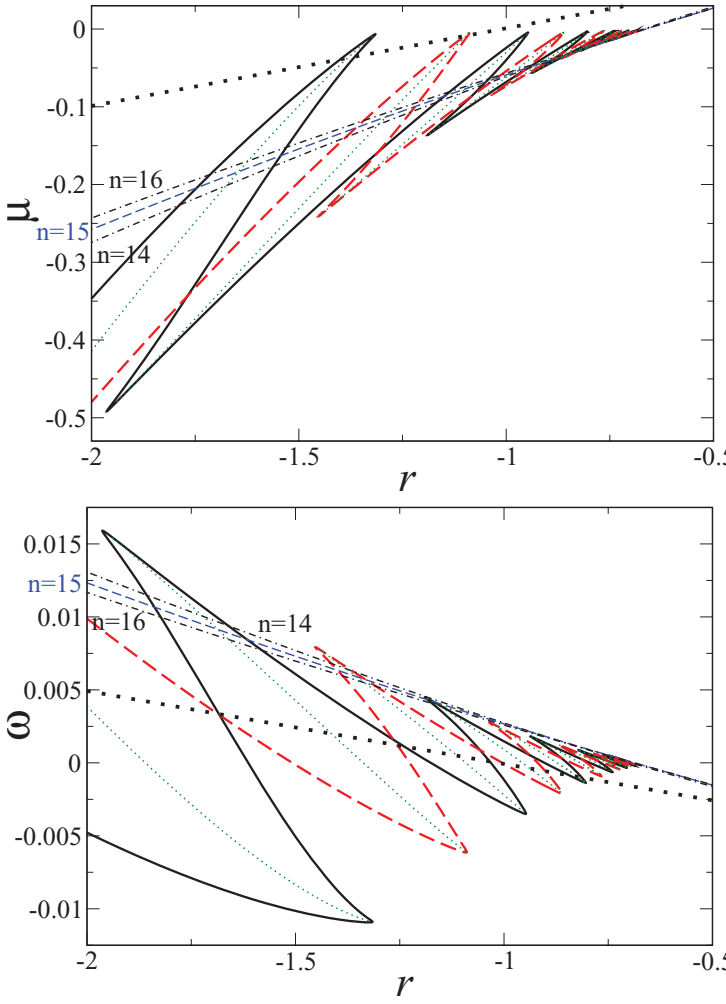

FIG. 3. (Color online) Bifurcation diagram showing localized solutions of the VPFC (an augmented conserved Swift-Hohenberg equation) [Eqs. (1) and (5)] with $H=1500$, as a function of the parameter $r$, for the mean order parameter $\bar{\phi}=0.1$ and a fixed domain size of $L=100$. The various solution profiles are characterized by their (a) $L^{2}$ norm, (b) chemical potential $\mu$, (c) mean free energy density $\left(F-F_{0}\right) / L$, and (d) mean grand potential density $\omega \equiv F / L-\bar{\phi} \mu$. The heavy black dotted line corresponds to the homogeneous solution $\phi(x)=\bar{\phi}$. Periodic solutions with $n=15$ bumps are shown as a thin blue dashed line, whereas the nearby thin black dash-dotted lines represent the $n=14$ and $n=16$ solutions as indicated in the plot. The heavy solid black and dashed red lines that bifurcate from the $n=15$ periodic solution represent symmetric localized states with a maximum (odd states) and a minimum (even states) at the center, respectively. The green dotted lines that connect the two branches of symmetric localized states correspond to asymmetric localized states. Together the branches of localized states form a slanted snakes-and-ladders structure.

indicating that in these cases the time simulation converges to a local and not the global energy minimum. This is to be expected as the solutions shown in the bifurcation diagrams are only the "tip of the iceberg." For instance, there exist many more solutions, where not all the inner distances between the bumps are identical. This is related to the fact that individual bumps have oscillatory tails and the "locking of these tails" allows for different equilibrium distances [24]. The solutions presented in Figs. 3 and 4 represent the solution having the lowest energy in the respective class. However, the energy differences between these and the "less symmetric" solutions are often tiny. Thus, it is not surprising that time simulations starting from random initial profiles often converge to solutions with greater disorder and energies than those shown in Fig. 4. For instance, the solution in Fig. 2(d) at $\bar{\phi}=0.1$ is a 9-bump solution similar to the odd symmetric localized states shown in the first two panels of the second row of Fig. 5. The amplitudes agree well and although the arrangements of the 9 bumps are different, the free energy and norm still agree to $<1 \%$. However, at large average order parameter values $\bar{\phi}$ the time simulation results can converge to metastable states with energies quite different from the minimum energy states for domains of this size $L=100$. For example, the periodic solution obtained from the time simulation [shown in Fig. 2(f)] when $\bar{\phi}=0.3$ has 18 bumps. However, from Fig. 4(c) we observe that the energetic minimum is obtained by a periodic profile with 15 bumps, as shown by the steady state solution in Fig. 5 . The convergence to a different number of bumps in the time simulation may be caused by discretization effects or by the initial noise profile used. As one would expect, the free energy associated with the 18-bump periodic structure is significantly larger than the 15-bump profile.

In Fig. $3(\bar{\phi}=0.1)$ the localized states bifurcate subcritically from the periodic solution branch (that itself emerges from the trivial homogeneous solution that is displayed as the heavy black dotted line). Therefore, one expects hysteretic behavior as encountered in the time simulations. A magnification (not shown) allows us to determine the threshold values for the hysteretic transition. When decreasing $r$ in the region where periodic solutions are always found, one first passes $r_{\mathrm{sn}}=-0.685$ where the last two branches of localized solutions annihilate in a saddle-node bifurcation [Fig. 3(a)]. Slightly below $r_{\mathrm{sn}}$, both the periodic solution and the localized state with a single bump are local energetic minima. Although the periodic solution represents the global minimum, particular time simulations sometimes converge to the localized state. 
(a)

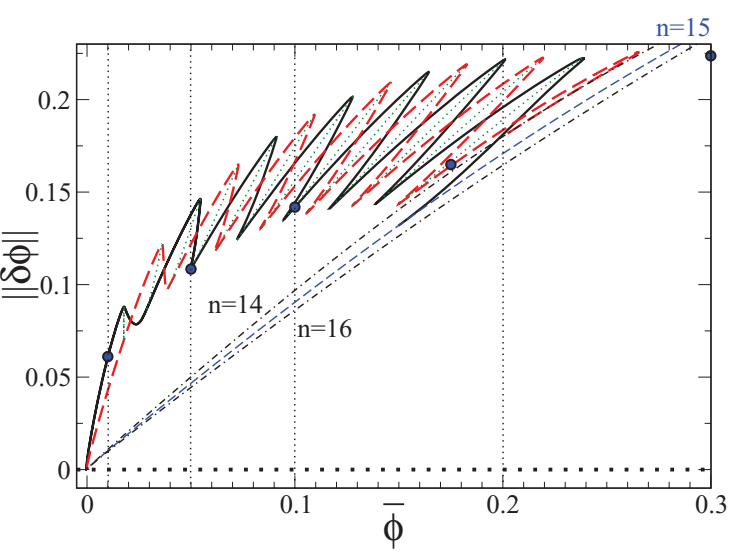

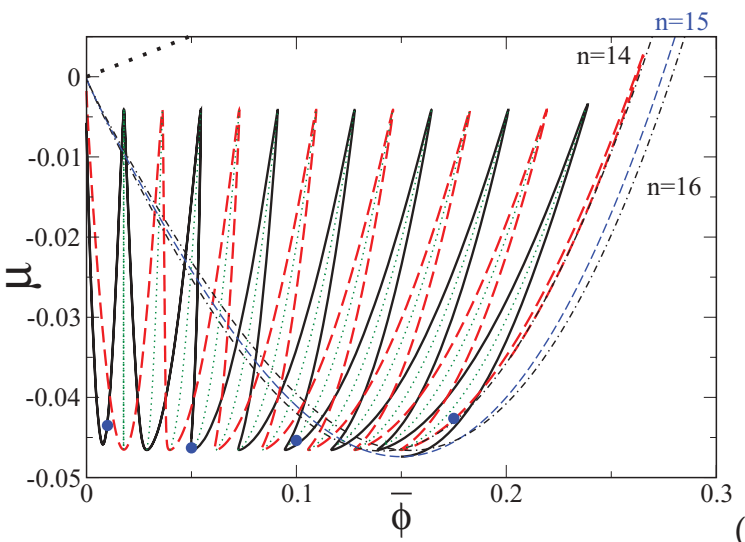
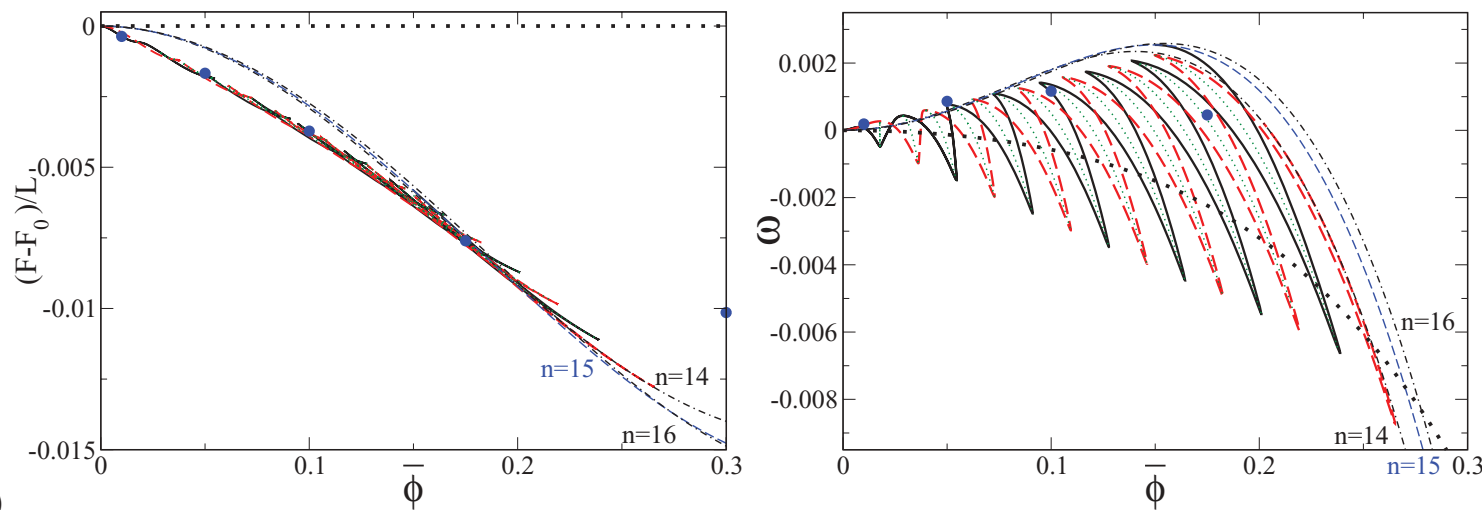

(d)

FIG. 4. (Color online) Bifurcation diagram showing localized solutions of the VPFC as a function of the mean order parameter $\bar{\phi}$, for $r=-0.9$ and a fixed domain size of $L=100$. The various solution profiles are characterized by their (a) $L^{2}$ norm, (b) chemical potential $\mu$, (c) mean free energy density $\left(F-F_{0}\right) / L$, and (d) mean grand potential density $\omega \equiv F / L-\bar{\phi} \mu$. The line styles are as in Fig. 3. Here, however, the heavy solid black and dashed red lines bifurcate at large $\bar{\phi}$ from the $n=15$ and $n=14$ periodic solutions, respectively. Typical profiles for all the branches of localized states are given in Fig. 5. The vertical dotted lines in (a) correspond to values of $\bar{\phi}$ for results in Fig. 5. The blue dots correspond to the five time simulation profiles shown in Figs. 2(b)-2(f).

The differences in energy between the two is $<1 \%$ in the case of Fig. 3. When $r$ is further decreased below $r_{\mathrm{en}}=-0.700$ the energy of the even symmetric states becomes smaller than the one of the $n=16$ periodic solution, which is, however, still linearly stable. The situation changes at $r_{\mathrm{c}}=-0.749$, where both symmetric localized branches bifurcate from the $n=16$ branch; that is, below $r_{\mathrm{c}}$ the latter is linearly unstable. Furthermore, below $r_{\mathrm{c}}$ the energy of all localized states rapidly becomes much smaller than the energy of all periodic states [Fig. 3(c)]. The hysteresis range displayed in Fig. 2 provides a good approximation for the region between $r_{\mathrm{c}}$ and $r_{\mathrm{sn}}$. This region becomes larger as $\bar{\phi}$ is increased.

The situation is very similar when $\bar{\phi}$ is changed for fixed $r$ (Fig. 4). The resulting hysteresis range is between $\bar{\phi}=0.150$ and 0.239 for symmetric localized states with an odd number of maxima and between $\bar{\phi}=0.202$ and 0.265 for symmetric states with an even number of maxima. Overall, one should therefore expect a wide hysteresis region roughly between $\bar{\phi}=0.15$ and $\bar{\phi}=0.25$. The hysteresis range obtained from the time simulations (indicated in Fig. 2) is roughly $0.19<$ $\bar{\phi}<0.22$. This is narrower than the range deduced from the path continuation analysis of the localized steady states, but lies right in the middle of it.

Before we move on to discuss the 2D case, we should comment on how our results fit into the wider context of research on localized states. Much research on localized states focuses on the nonconserved Swift-Hohenberg equation [19-21]. There, such states can exist only if the primary bifurcation of periodic states from the homogeneous base state is subcritical. The localized states exist in a subrange of the existence range of the periodic states bounded on either side by the saddlenode bifurcations of the branches of symmetric localized states. In the nonconserved Swift-Hohenberg equation these accumulate exponentially rapidly toward the parameter values corresponding to first and last tangencies between the unstable manifold of the homogeneous state in space and the stable manifold of the periodic state. These tangencies define the pinning region containing the different localized structures. In contrast, in the presence of a conserved quantity localized states may exist outside the existence region of periodic states and may occur even in the supercritical case, and the saddle-node bifurcations of the localized states are no longer aligned; that is, one finds slanted snaking [25]. This is typically a finite size effect [26].

For the regular PFC (conserved Swift-Hohenberg equation) [Eq. (1) with Eqs. (2) and (3)], localized states are briefly mentioned in Ref. [8]. However, no systematic results along the lines of those presented in Refs. [19] for nonconserved or [25] for conserved order parameter fields are available. The model used here is a special case because it includes the 


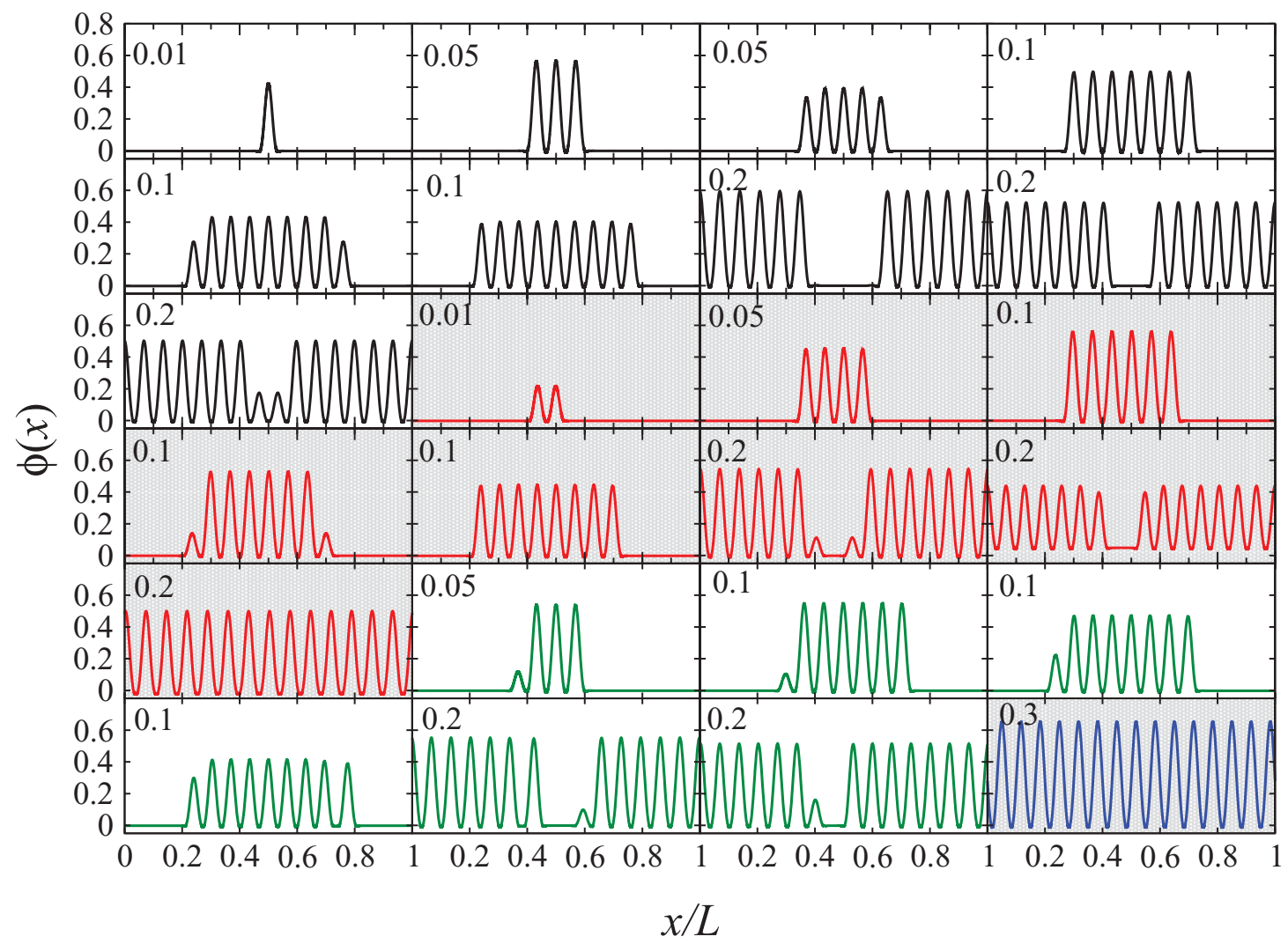

FIG. 5. (Color online) A selection of steady state profiles $\phi(x)$ for $r=-0.9$ at $\bar{\phi}=0.01,0.05,0.1$, and 0.2 . From top left to bottom right we show first nine type (i) solutions, that is, symmetric localized states with an odd number of maxima (in black), then eight type (ii) solutions, that is, symmetric localized states with an even number of maxima (in red), followed by six type (iii) solutions, that is, asymmetric localized states (in green). The final image is the $n=15$ periodic solution at $\bar{\phi}=0.3$ (in blue). The number in each panel indicates the corresponding value of $\bar{\phi}$. The solutions from the symmetric branches are shown in the sequence that follows the respective branch in Fig. 4(a), starting from the left. The asymmetric states for identical $\bar{\phi}$ are shown in the order of decreasing norm.

nonanalytic vacancy term (5). However, a similar bifurcation structure is found for the classical conserved Swift-Hohenberg equation, that is, the regular PFC model. We will present results from our study of localized states in the regular PFC model elsewhere [27].

\section{Two-dimensional model}

We now move on to consider how the VPFC model behaves in 2D. As with the regular PFC model [2], when we expand into 2D we observe stripes [see Fig. 6(b)] and hexagonally ordered bumps or holes [see Fig. 6(c)]. In Fig. 6(a) we display the phase diagram of the VPFC model in 2D and typical time simulation results from the striped [Fig. 6(b)] and hole [Fig. 6(c)] phases, calculated on a regular grid with grid spacing $d x=0.5$. Square ordering of bumps or holes does not appear in the phase diagram because these structures always have a higher free energy. However, this can be changed through appropriate alterations to the free energy [5]. Square ordering can also occur when extending to a two-component mixture (cf. Sec. III C below). Using the same method as outlined above, we calculate the regions of the phase diagram where there is coexistence between hexagonally ordered holes and the uniform distribution, between holes and stripes, and between stripes and hexagonally ordered bumps. The vacancy term (5) shifts the modulated phases into the positive $\bar{\phi}>0$ plane. The section of the phase diagram where holes are observed is much smaller when compared to the regular PFC model and now extends beyond the limit of linear stability of the flat state (at $\Delta=0$ ). This means that for certain values of $\bar{\phi}$ (where $0<\Delta \ll 1$ ), hexagonally arranged holes are energetically favorable but are only observed in time simulations for certain initial conditions; that is, when starting with an order parameter profile $\phi(\mathbf{x}, t=0)$ which already has modulations which are sufficiently large in amplitude. As $r$ is decreased (i.e., for larger $|r|$ ) it becomes increasingly difficult to obtain structures with holes up to and inside of the coexistence region between the hole and the uniform phases. This is a direct consequence of the limit of linear stability occurring in the middle of the hole phase. Therefore, the accuracy of results for the coexistence region between the hole and uniform phases decreases as $|r|$ becomes larger. The stripe phase occurs between the two hexagonal phases. In the order parameter profiles displayed in Figs. 6(b) and 6(c) we observe various defects and in Fig. 6(c) "grain" boundaries between regions with different orientations, which depend on the initial conditions (our initial profile was a flat state with additional small amplitude white noise). The true minimum profile for case (b) is a series of parallel stripes which are identical to the periodic profiles in the 1D system [shown in Fig. 2(f)]. 
(a)

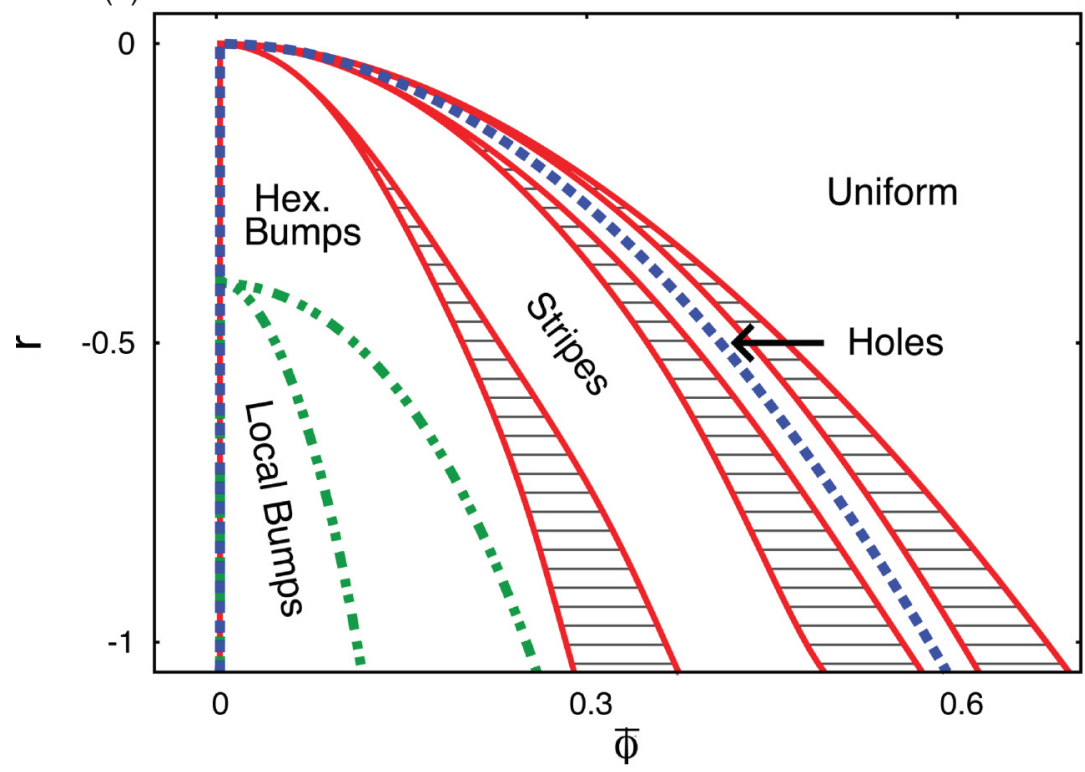

(b)

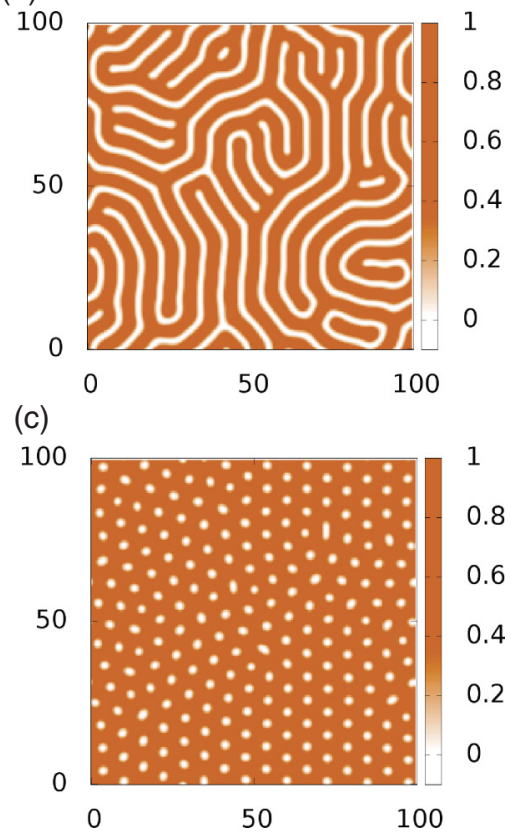

FIG. 6. (Color online) The phase diagram for the 2D VPFC model [Eqs. (1) and (4)] is displayed in (a) for the case $q=1$. The red solid lines are the coexistence curves between the various phases. The blue dashed line is the limit of linear stability for uniform profiles $\Delta=0$. The green dash-dotted lines indicate the region where localized and hexagonally ordered bump structures coexist. Simulations of (b) stripes and (c) hexagonally ordered holes are also shown. The parameter values for these simulations are $q=1, r=-0.9, \alpha=1$ and (b) $\bar{\phi}=0.4$ and (c) $\bar{\phi}=0.53$.

The most important portion of the phase diagram from the materials modeling point of view, is the bump phase because the basic assumption is that each bump represents a particle. When $r \gtrsim-0.4$ or when $\bar{\phi}$ has a value close to that in the coexistence region between bumps and stripes, we observe hexagonally arranged bumps, similar to those in the regular PFC model. However, in a manner similar to the 1D system, we observe localized structures at small values of $\bar{\phi}$ when $r \lesssim$ -0.4 . In the phase diagram [Fig. 6(a)] the green dot-dashed lines are numerically obtained estimates for the location in the phase diagram of the limits of linear stability of the uniform periodic states (lower curve) and the localized (vacancy) states (upper curve). They are determined in the same manner as discussed above for the 1D system for a square system of side length $L=25$. It is important to note that the parameter range where localized bumps coexist with regular periodically ordered bumps is much broader for the 2D system, implying a large amount of hysteresis.

We now focus our discussion on the portion of the phase diagram where isolated bumps form. As can be seen in Fig. 7, these profiles resemble particle configurations in gases, liquids and crystalline solids and so the VPFC may be a valuable model for describing materials on the microscale [13]. This region of the phase diagram is full of complexity and many varied structures may be observed. However, here we forego a full systematic study of this large region in parameter space and limit ourselves to showing representative results obtained for a single value of the undercooling parameter $r=-0.9$ for which there is a fairly large range in $\bar{\phi}$ with isolated bumps. We set the initial order parameter profile to be a uniform state with a small amplitude noise $\phi(\mathbf{x}, t=0)=\bar{\phi}+\lambda(Y-0.5)$, where $Y$ is a random real number uniformly distributed between 0 and 1 and $\lambda=10^{-6}$ is the amplitude of the noise. We consider three cases: (i) $\bar{\phi}=0.01$ and (ii) $\bar{\phi}=0.1$, where a disordered arrangement of localized bumps forms, and (iii) $\bar{\phi}=0.24$, which is in the region where bumps are hexagonally ordered. We average over many simulations to calculate the two-point correlation function for each of these cases. This is done by locating all the maxima in the equilibrium profile $\phi(\mathbf{x})$, for a given initial realization of the noise; that is, we locate the position of all the bumps. From these sets of coordinates we calculate the radial distribution function $g(x)$ in the usual way [28]. We display a simulation result for case (i) in Fig. 7(a) and the corresponding radial distribution function $g(x)$ in Fig. 7(d). We find that there is almost no correlation between the bumps in this circumstance except for the core repulsion and a very small peak at $x \approx 16$, indicating that there is a weak attraction between the bumps. Therefore, simulations with these parameter values appear to qualitatively describe gaslike formations of particles or colloids. In Figs. 7(b) and 7(e) we plot a typical order parameter profile and the corresponding $g(x)$ for case (ii). We observe a large increase in the number of bumps as compared to the previous case. The radial distribution function shows that we have strong short range ordering, but without any long range order. This is very reminiscent of the ordering in liquids. There is a very sharp peak in $g(x)$ at around $x=7.5$ (which is approximately the diameter of the bumps) and a smaller peak around $x=15$. A similar example is also given in Ref. [13]. If we further increase the value of $\bar{\phi}$ we eventually find the more familiar hexagonally structured array of bumps which is reminiscent of the ordering in simple crystalline solids. In Fig. 7(c) we display an example of the order parameter profile for case (iii) and in Fig. 7(f) we show the corresponding $g(x)$. For 
(a)

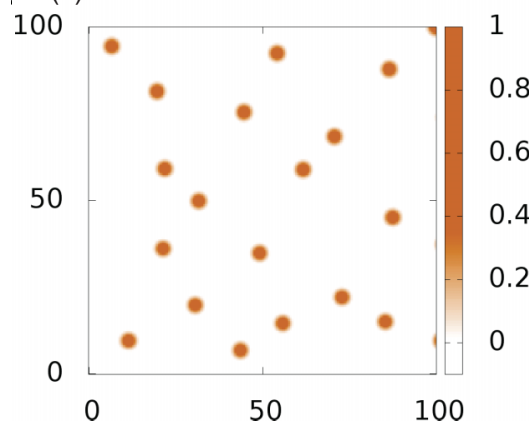

(d)

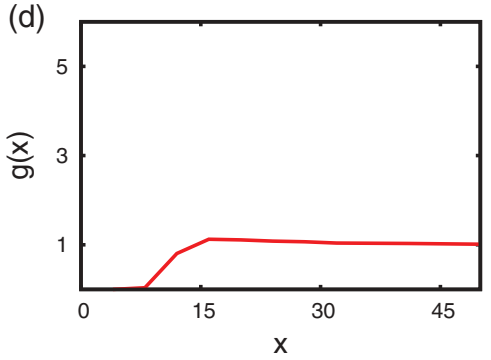

(b)

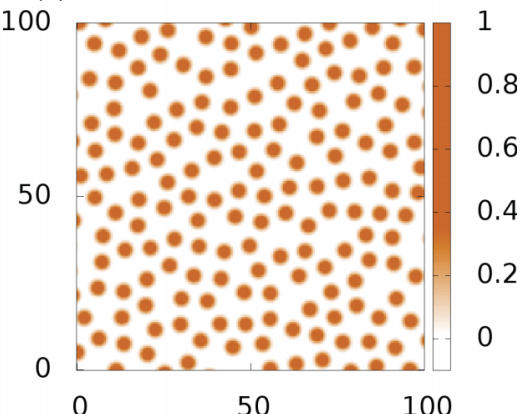

(e)

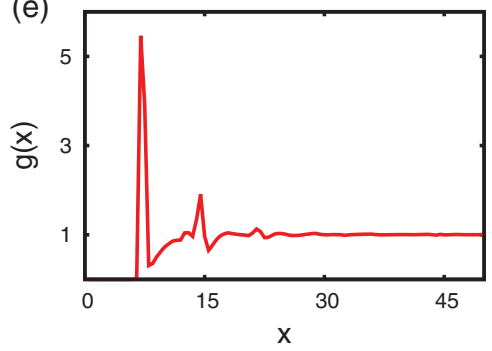

(c)

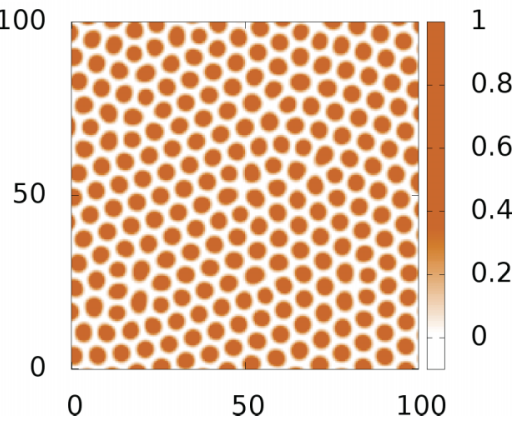

(f)

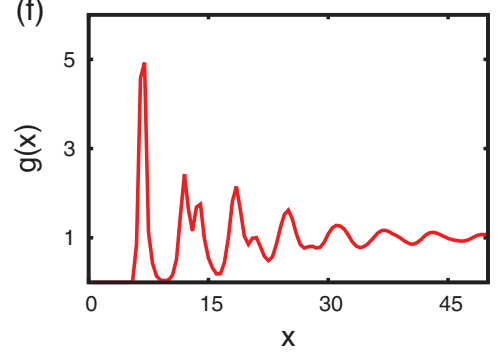

FIG. 7. (Color online) (a)-(c) Typical steady state order parameter profiles obtained in time simulations for increasing $\bar{\phi}$. (d)-(f) The corresponding radial distribution function $g(x)$ calculated from multiple simulations. The parameter values are $\alpha=1, q=1, r=-0.9$ and in (a), (d) $\bar{\phi}=0.01$, in (b), (e) $\bar{\phi}=0.1$, and in (c),(f) $\bar{\phi}=0.24$.

this case we observe that $g(x)$ is highly structured, indicating the system has very strong short range correlations with a significant degree of long range ordering. We observe the split second and third peaks, which is a classic sign of crystalline order. These results indicate that the VPFC model may be used to model crystalline structures, much like the regular PFC model. The major difference between the two models is the existence of the fluidlike configuration of bumps observable in the VPFC model. In contrast, the fluid phase in the PFC model corresponds to the homogeneous state.

The variation in the size and shape of the bumps that are formed is fairly small. In Fig. 8(a) we display a selection of results for the order parameter profile through the center of the bumps for the case when $r=-0.9$ and $\bar{\phi}=0.01$. We determine the shape of the bumps by plotting the value of the order parameter $\phi$ against the distance from the peak of each bump (as shown by the data points). We can then fit functions which take the following form:

$$
\theta(x)=\beta_{0} e^{-\beta_{1} x^{2}-\beta_{2} x^{4}-\beta_{3} x^{6}} \cos \left(\beta_{4} x\right)+\beta_{5} .
$$

We fit this form to the data using a least squares method. The exponential part of $\theta(x)$ describes the decay of the modulation as the distance from the peak increases and the cosine function captures the oscillatory tail of the modulations, which is an important factor in their interaction with other bumps [29,30]. Figure 8(a) displays two cases: the + points and red solid line show the case $q=1$ and the $\times$ points and blue dashed line show the case where $q=1.1$. The size of the bump is reduced as we increase the value of $q$. This is because increasing the value of $q$ increases the typical wave number, which results in a smaller typical length scale [cf. Fig. 1 and Eq. (10)].

The curves obtained from fitting the bump profile can be used to obtain an approximation for the effective pair potential $V(x)$ between two isolated bumps, where $x$ is the distance between the centers of the bumps. We take a uniform system with the value of $\phi$ equal to that in the uniform areas between bumps found in simulations for $\bar{\phi}=0.01$, corresponding to the results in Fig. 7(a). We then impose upon this the profiles for two bumps using the fitted curves shown in Fig. 8(a). We vary the distance between the superposed bumps and calculate the free energy of the system. We assume thereby that the two bumps retain their shape when they are close, despite the fact that, in reality, the bump shapes become distorted as bumps are pushed close together.

In Fig. 8(b) we display the results for $q=1$ (red solid line) and $q=1.1$ (blue dashed line). We observe that there is a shallow minimum in the potential at the distance $x \approx 7.5$ when $q=1$ and at $x \approx 7$ when $q=1.1$ [see inset of Fig. 8(b)]. The minimum is at a smaller distance when $q$ is larger because of the decreased diameter of the bumps; recall that $q$ determines the size of the bumps. The resulting weak attraction between the bumps may also be inferred from the radial distribution function $g(x)$ calculated for the low density case $\bar{\phi}=0.01$ when $q=1$ displayed in Fig. $7(\mathrm{~d})$. We observe a second minimum in the potentials at $x \approx 3.15$ when $q=1$ and at $x \approx 2.3$ when $q=1.1$, where the former rather appears like a "shoulder." The order parameter profiles at the second minima resemble the elongated almost elliptical shapes which are observed in and around the coexistence region between bumps and stripes. See also Fig. 6(c) where we also observe elliptical holes along some of the grain boundaries.

\section{TWO-COMPONENT SYSTEM}

We now extend the model to consider a binary mixture in perhaps the most simple way possible, by adding together free energies like Eq. (4) for two order parameter fields $\phi_{a}(\mathbf{x}, t)$ and $\phi_{b}(\mathbf{x}, t)$. We introduce a simple coupling term which allows 

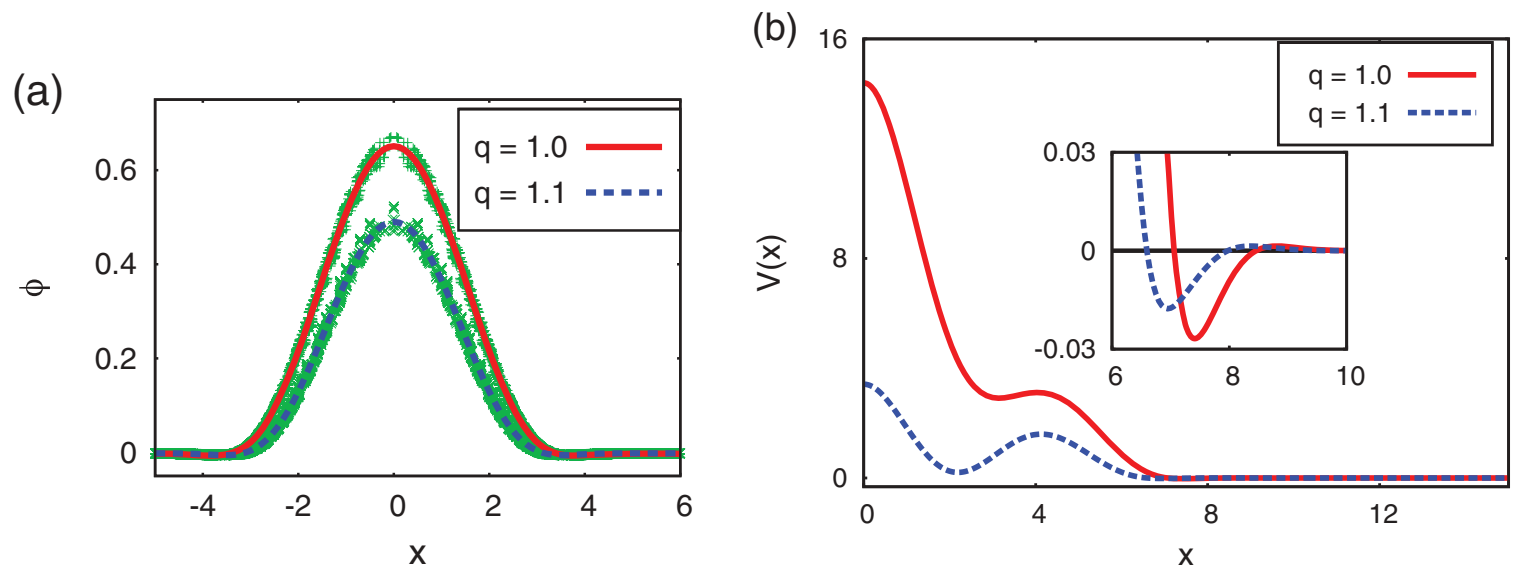

FIG. 8. (Color online) (a) Several sets of numerical results for the order parameter profile through the center of a bump ( + ) for $q=1$ and $(\times)$ for $q=1.1$, together with fits to the data displayed as solid red and dashed blue lines. These fits are then used to calculate the effective pair potential between two bumps. These pair potentials are displayed in (b). The inset displays a magnification of the tails of $V(x)$. The parameter values are $r=-0.9$ and $\bar{\phi}=0.01$.

the two components to interact with each other. This gives us the following expression for the free energy:

$$
\begin{aligned}
F= & \int d \mathbf{x}\left[f\left(\phi_{a}(\mathbf{x}, t)\right)+f_{\mathrm{vac}}\left(\phi_{a}(\mathbf{x}, t)\right)+f\left(\phi_{b}(\mathbf{x}, t)\right)\right. \\
& \left.+f_{\mathrm{vac}}\left(\phi_{b}(\mathbf{x}, t)\right)+\eta \phi_{a} \phi_{b}\right]
\end{aligned}
$$

where $\eta$ is the coupling coefficient and the functions $f$ and $f_{\text {vac }}$ are defined as before in Eqs. (3) and (5). The value of $r$ is set equal for both components. However, we allow the value of $q$ to be different for each species, so we now refer to these values as $q_{a}$ and $q_{b}$, where the subscript denotes the corresponding component. Setting different values for $q$ in the two components (i.e., $q_{a} \neq q_{b}$ ) results in an asymmetric system in which the size of the bumps or modulations in $\phi_{a}(\mathbf{x})$ differs from that in $\phi_{b}(\mathbf{x})$, as discussed further below in Secs. III B and III C. Note that a different coupling term is used in Ref. [14]; a somewhat different two-component PFC model is presented in Ref. [31]. In our two-component VPFC model one may interpret the bumps in the profile $\phi_{a}(\mathbf{x})$ as representing particles of species $a$ and the bumps in the other profile $\phi_{b}(\mathbf{x})$ as representing particles of species $b$. There are also vacant "vacancy" areas, where both order parameter profiles take values close to zero.

Here, just as for the one-component model, we assume the dynamics of the system is governed by the following pair of equations [cf. Eq. (1)]:

$$
\frac{\partial \phi_{a}}{\partial t}=\alpha_{a} \nabla^{2} \frac{\delta F}{\delta \phi_{a}}, \quad \frac{\partial \phi_{b}}{\partial t}=\alpha_{b} \nabla^{2} \frac{\delta F}{\delta \phi_{b}} .
$$

We also assume that the two mobility coefficients are equal: $\alpha_{a}=\alpha_{b}=\alpha$. The two components are coupled purely by the term $\eta \phi_{a} \phi_{b}$ in the free energy. When $\eta>0$, this coupling term leads to a repulsion between the two species and so penalizes structures which overlap or form on top of each other. The value of the parameter $\eta$ determines the "strength" of the coupling, and so the two-component model reduces to two disconnected one-component models in the limit $\eta \rightarrow 0$.

\section{A. Phase behavior}

When the coupling coefficient is fairly large, $\eta \geqslant 0.1$, the coupling term has a significant impact on the phase behavior of the model. In particular, the limit of linear stability and the phase coexistence curves extend to much larger values of $\bar{\phi}=\bar{\phi}_{a}+\bar{\phi}_{b}$ than for the one-component model. We now determine the linear stability of a flat state in the model. We assume that the order parameter profiles of both components take the form

$$
\begin{aligned}
& \phi_{a}=\bar{\phi}_{a}+\delta \phi=\bar{\phi}_{a}+\xi e^{i k \mathbf{x}} e^{\beta t}, \\
& \phi_{b}=\bar{\phi}_{b}+\chi \delta \phi=\bar{\phi}_{b}+\chi \xi e^{i k \mathbf{x}} e^{\beta t},
\end{aligned}
$$

where the amplitude $|\xi| \ll 1$ and the parameter $\chi$ is the ratio between the amplitude of the modulations in the two components. The sign of $\chi$ indicates whether instabilities are in phase $(\chi>0)$ or antiphase $(\chi<0)$ between the two coupled order parameter fields. From the magnitude of $\chi$ we can deduce whether the instability is initiated from species $a(|\chi| \ll 1)$, species $b(|\chi| \gg 1)$, or a combination of both $(|\chi|=O(1))$. We make a Taylor series expansion of the functional derivatives of the free energy with respect to the two order parameters $\phi_{a}$ and $\phi_{b}$, to obtain

$$
\begin{aligned}
\frac{\delta F}{\delta \phi_{a}}= & \left(r+q_{a}^{4}\right) \bar{\phi}_{a}+3 H \bar{\phi}_{a}\left(\left|\bar{\phi}_{a}\right|-\bar{\phi}_{a}\right)+\bar{\phi}_{a}^{3}+\eta \bar{\phi}_{b} \\
& +\left[\left(k^{2}-q_{a}^{2}\right)^{2}+\Delta_{a}+\chi \eta\right] \delta \phi+O\left(\delta \phi^{2}\right), \\
\frac{\delta F}{\delta \phi_{b}}= & \left(r+q_{b}^{4}\right) \bar{\phi}_{b}+3 H \bar{\phi}_{b}\left(\left|\bar{\phi}_{b}\right|-\bar{\phi}_{b}\right)+\bar{\phi}_{b}^{3}+\eta \bar{\phi}_{a} \\
& +\left[\chi\left(k^{2}-q_{b}^{2}\right)^{2}+\chi \Delta_{b}+\eta\right] \delta \phi+O\left(\delta \phi^{2}\right),
\end{aligned}
$$

where $\Delta$ is defined as before Eq. (8) and the subscript denotes the corresponding component. We substitute these expressions into the dynamical equations (23), yielding the matrix problem $[32,33]$

$$
\beta\left(\begin{array}{l}
1 \\
\chi
\end{array}\right)=\mathbf{M}\left(\begin{array}{l}
1 \\
\chi
\end{array}\right)
$$


where

$$
\mathbf{M}=-k^{2} \alpha\left(\begin{array}{lc}
\left(q_{a}^{2}-k^{2}\right)^{2}+\Delta_{a} & \eta \\
\eta & \left(q_{b}^{2}-k^{2}\right)^{2}+\Delta_{b}
\end{array}\right) .
$$

We can now determine the dispersion relation $\beta(k)$ by calculating the eigenvalues of $\mathbf{M}$ :

$$
\beta(k)=\frac{\operatorname{Tr}(\mathbf{M})}{2} \pm \sqrt{\frac{\operatorname{Tr}(\mathbf{M})^{2}}{4}-|\mathbf{M}|} .
$$

The resulting dispersion relation $\beta(k)$ is a double-valued function. However, since the growth rate along the + branch is always larger than that along the - branch, the limit of linear stability can be determined from the + branch alone. If we assume that $q_{a}=q_{b}=q$, the dispersion relation simplifies significantly, yielding

$$
\begin{aligned}
\beta(k)= & -\frac{\alpha k^{2}}{2}\left[2\left(k^{2}-q^{2}\right)^{2}+\Delta_{a}+\Delta_{b}\right. \\
& \left.-\sqrt{\left(\Delta_{a}-\Delta_{b}\right)^{2}+4 \eta^{2}}\right] .
\end{aligned}
$$

There is a local maximum of this expression which occurs at the positive wave number:

$$
\begin{aligned}
k_{m}= & \frac{1}{6}\left[24 q^{2}+6\left(4 q^{4}-6\left(\Delta_{a}+\Delta_{b}\right)\right.\right. \\
& \left.\left.+6 \sqrt{\left(\Delta_{a}-\Delta_{b}\right)^{2}+4 \eta^{2}}\right)^{\frac{1}{2}}\right]^{\frac{1}{2}} .
\end{aligned}
$$

Substituting this wave number back into the dispersion relation (28), allows us to calculate the parameter values such that $\beta\left(k_{m}\right)=0$ (i.e., the limit of linear stability of a flat state). We arrive at the following relation:

$$
\Delta_{a} \Delta_{b}=\eta^{2} \text {. }
$$

When the system is linearly unstable it is possible for $\beta(k=$ $0)$ to be a minimum or maximum (this transition occurs at $\Delta=-q^{2}$ in the one-component model). This is equivalent to the coefficient of $k^{2}$ changing from a positive value (minimum) to a negative value (maximum). The sign of the coefficient of $k^{2}$ is determined by the sign of the following quantity:

$$
\begin{aligned}
C_{2} & =\frac{\partial^{2} g}{\partial \phi_{a}^{2}} \frac{\partial^{2} g}{\partial \phi_{b}^{2}}-\left(\frac{\partial^{2} g}{\partial \phi_{b} \partial \phi_{a}}\right)^{2}, \\
& =\left(q_{a}^{4}+\Delta_{a}\right)\left(q_{b}^{4}+\Delta_{b}\right)-\eta^{2},
\end{aligned}
$$

where $\quad g\left(\phi_{a}, \phi_{b}\right)=f\left(\phi_{a}\right)+f_{\mathrm{vac}}\left(\phi_{a}\right)+f\left(\phi_{b}\right)+f_{\mathrm{vac}}\left(\phi_{b}\right)+$ $\eta \phi_{a} \phi_{b}$. When $C_{2}$ is negative (positive) $\beta(k=0)$ is a minimum (maximum); this relation also holds for asymmetric systems where $q_{a} \neq q_{b}$. In Fig. 9 we display typical dispersion relations when $q_{a}=q_{b}=1, \Delta_{a}=\Delta_{b}=\Delta$, and $\eta=4$. We show the case when (i) the system is linearly unstable and $C_{2}$ [Eq. (31)] is negative (red solid line), (ii) the system is linearly unstable and Eq. (31) is positive (blue dashed line), (iii) the system is at the limit of linear stability [i.e., Eq. (30) holds] (green dotted line), and (iv) the system is linearly stable (magenta dash-dotted line). We observe that when $q_{a}=q_{b}$, the typical wave number $k_{m} \rightarrow q_{a}$ as we approach the limit of linear stability $\Delta_{a} \Delta_{b}-\eta^{2} \rightarrow 0$. In the more general case

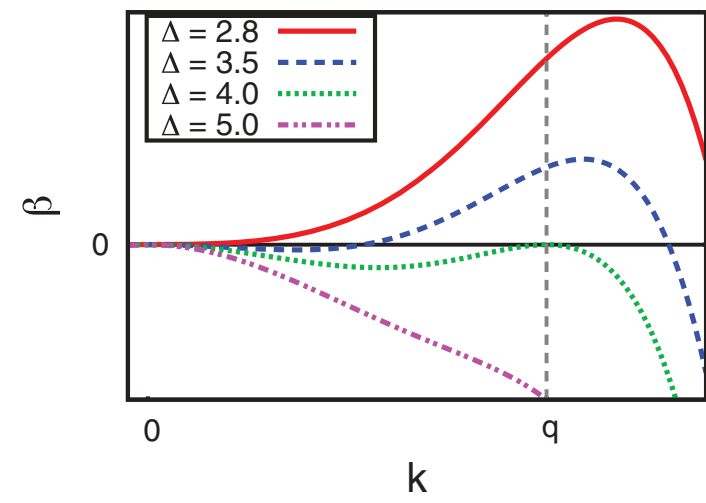

FIG. 9. (Color online) Dispersion curves for the two-component VPFC model [Eqs. (22) and (23)], when $q_{a}=q_{b}=1, \Delta_{a}=\Delta_{b}=\Delta$, and $\eta=4$. Four cases are shown: (i) $\beta\left(k_{m}\right)>0$ with $\beta(k=0)$ a minimum (red solid line), (ii) $\beta\left(k_{m}\right)>0$ and $\beta(k=0)$ a maximum (blue dashed line), (iii) $\beta\left(k_{m}\right)=0$ (green dotted line), and (iv) $\beta\left(k_{m}\right)<0$ (magenta dash-dotted line).

with $q_{a} \neq q_{b}$ the dispersion relation may have two maxima at positive values of $k$, neither of which occurs at $q_{a}$ and $q_{b}$. In this case the stability boundary is defined by the vanishing of the growth rate $\beta\left(k_{m}\right)=0$ of the larger of the two possible maxima of $\beta$.

From Eq. (30) it is clear that depending on the value of the coupling coefficient $\eta$, the region of parameter space where the system is linearly unstable can be greatly larger than that for the one-component system. For example, picking the value $\eta=4$ when $r=-0.9$ and setting the average value of both order parameters to be equal $\bar{\phi}_{a}=\bar{\phi}_{b}=\bar{\phi}$, we find that the limit of linear stability increases from $\bar{\phi}=0.548$ (for the one-component case) to $\bar{\phi}=1.278$. As one would expect, this also increases the region of the phase diagram where modulated structures are formed. Our focus here is on the regions of parameter space where bumps are formed as this is the regime relevant to modeling crystalline solids. However, before proceeding to this, we make a brief survey of some of the structures which may be observed for larger values of $\phi_{a}$ and $\phi_{b}$ which lie outside of the bump phase. For the parameter values $r=-0.9, \eta=4, q_{a}=q_{b}=1$, and $\bar{\phi}_{a}=\bar{\phi}_{b}=\bar{\phi}$ we show in Fig. 10 a sequence of order parameter profiles with increasing $\bar{\phi}$, for values of $\bar{\phi}$ that lie above the region where bumps are observed (see later sections for a detailed analysis of the bump structures found in the two-component model). In Fig. 10 we display scaled plots of order parameter profiles which are stationary states obtained from time simulations. We plot an order parameter defined as the normalized difference between the $\phi_{i}(\mathbf{x})$ values of the two components $\Delta \hat{\phi}(\mathbf{x}) \equiv \phi_{a}(\mathbf{x}) / \hat{\phi}_{a}-\phi_{b}(\mathbf{x}) / \hat{\phi}_{b}$, where

$$
\hat{\phi}_{i}=\frac{\phi_{a}^{\max } \phi_{b}^{\max }-\phi_{a}^{\min } \phi_{b}^{\min }}{\phi_{i}^{\max }+\phi_{i}^{\min }}
$$

and where $\phi_{i}^{\max }$ and $\phi_{i}^{\min }$ are, respectively, the maximum and minimum values of $\phi_{i}(\mathbf{x}) . \Delta \hat{\phi}$ is defined so as to take a value in the range $[-1,1]$. When $\Delta \hat{\phi} \approx+1$, then the local value of $\phi_{a}$ is high while the value of $\phi_{b}$ is low. Conversely, when $\Delta \hat{\phi} \approx-1$, then the local $\phi_{a}$ is low and $\phi_{b}$ is high. The 


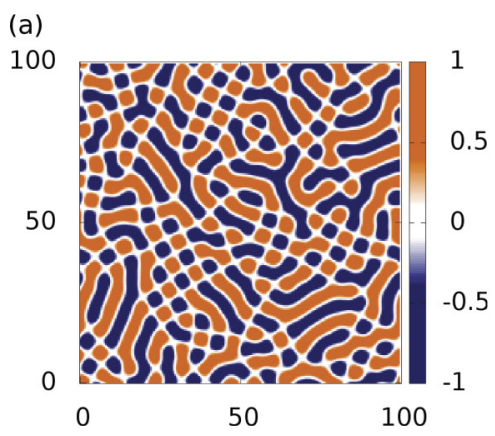

(d)

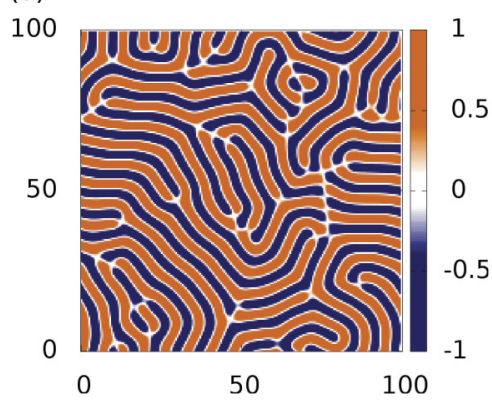

(b)

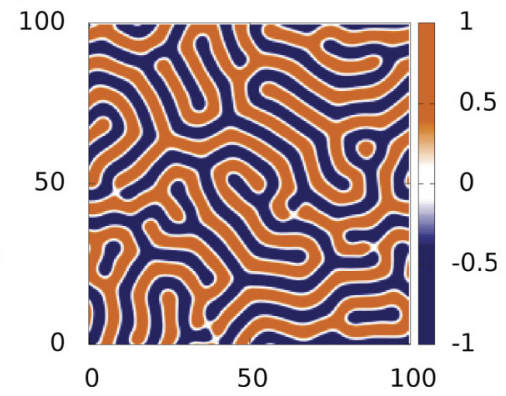

(e)

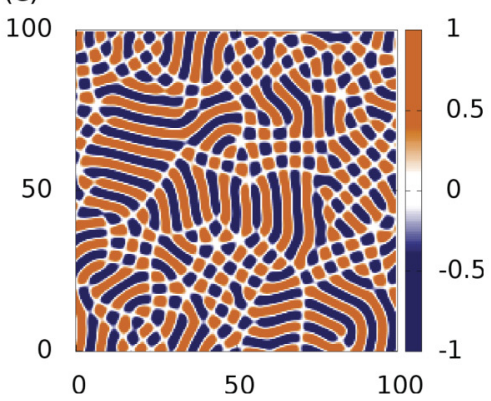

(c)

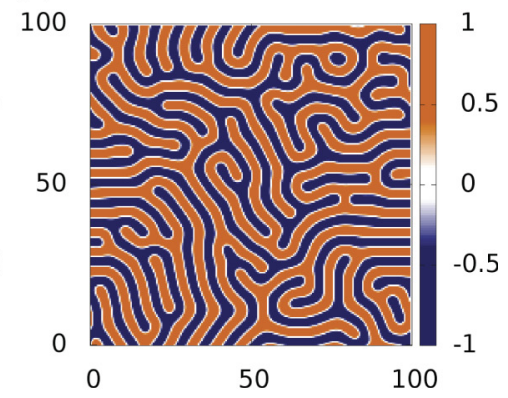

(f)

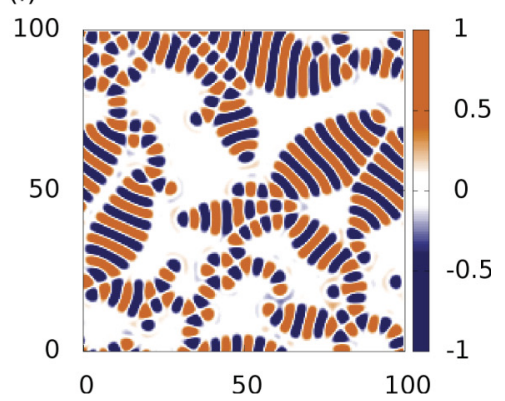

FIG. 10. (Color online) The scaled order parameter $\Delta \hat{\phi}$ for the two-component model, corresponding to minima of the free energy. The peaks in species $a$ are shown in orange (light gray), peaks in species $b$ are shown as blue (dark gray), and white areas show regions where $\phi_{a} \approx \phi_{b}$. The parameter values are $\eta=4, r=-0.9, q_{a}=q_{b}=1, \bar{\phi}_{a}=\bar{\phi}_{b}=\bar{\phi}$, where (a) $\bar{\phi}=0.25$, (b) $\bar{\phi}=0.3$, (c) $\bar{\phi}=1$, (d) $\bar{\phi}=1.15$, (e) $\bar{\phi}=1.2$, and (f) $\bar{\phi}=1.27$.

average order parameter values in Fig. 10 are (a) $\bar{\phi}=0.25$, (b) $\bar{\phi}=0.3$, (c) $\bar{\phi}=1$, (d) $\bar{\phi}=1.15$, (e) $\bar{\phi}=1.2$, (f) $\bar{\phi}=1.27$. The most palpable change from the one-component model is that the phase diagram is largely dominated by the striped profiles, with stripes appearing in the range $0.22 \lesssim \bar{\phi} \lesssim 1.28$. Just outside the range of $\bar{\phi}$ where bump structures are formed, we observe order parameter profiles which contain a mixture of bumps and stripes [see Fig. 10(a)]; this value of $\bar{\phi}$ must lie inside the coexistence region between the bump and stripe phases. As we increase the value of $\bar{\phi}$ we enter the large region of parameter space where stripe structures are formed [Figs. 10(b) and 10(c)]. The only significant change as we increase $\bar{\phi}$ from 0.3 to 1 is the decrease in the width of the stripes; this is due to the fact that the typical length scale in the system is $2 \pi / k_{m}$, where the wave number $k_{m}$ given by Eq. (29) is inversely proportional to the average order parameter values $\bar{\phi}_{a}$ and $\bar{\phi}_{b}$. Increasing the value of $\bar{\phi}$ further, we continue to observe striped profiles, but now there are points where the stripes of one species "connect" to stripes of the other species [see Fig. 10(d), where these "connections" appear as white lines]. Increasing $\bar{\phi}$ further, we observe a mixture of holes and stripes [Fig. 10(e)]. Close to the instability curve (30) we find interesting profiles where we observe a mixture of stripes, holes, and regions where the profile is approximately uniform $\phi_{a} \approx \phi_{b} \approx \bar{\phi}$ [Fig. 10(e)]. Various modulated structures are observed over a large range of parameter values. It would be possible to consider the structures formed for different values of the coupling coefficient $\eta$ and different values of the average order parameters, where $\bar{\phi}_{a} \neq \bar{\phi}_{b}$. However, here we do not make a systematic study of the entire parameter space and instead focus on the various bump configurations. These structures closely resemble the configurations of parti- cles or colloids in condensed matter systems and we believe that in this regime the model may be useful in understanding the fluid and solid phases of such systems.

\section{B. Intermolecular interactions}

For the remainder of this paper, we pursue the idea that the bumps in this two-component model represent two different types of molecules or colloidal particles suspended in a fluid medium. We perform time simulations of the two-component model choosing parameter values which result in the formation of bump structures. We run these simulations until the order parameter profiles reach an (almost) stationary state, which corresponds to being at (near) an energetic minimum. We then determine the coordinates of the particles by locating the position of the maximum of each of the peaks. The radial distribution functions are calculated by analyzing these coordinates. We also calculate the effective pair potentials between the bumps. Later in Sec. IIIC we consider the nearest neighbor bond angles and the ordering in crystalline configurations.

The bump phase in the two-component model appears to behave in a manner similar to that of the one-component model (cf. Fig. 6). We observe bump structures when the average value of the order parameters $\bar{\phi}_{a}$ and $\bar{\phi}_{b}$ are small. In particular, when $\bar{\phi}_{a}=\bar{\phi}_{b}=\bar{\phi}$ and $r=-0.9$ we observe bumps within the range $0 \lesssim \bar{\phi} \lesssim 0.15$. We study and compare two different systems: the symmetric case where $q_{a}=q_{b}=1$ and the asymmetric case where $q_{a}=1$ and $q_{b}=1.1$. In the symmetric case, interactions between bumps of the same type $(a a$ and $b b)$ are identical in both components, but the nature of the interaction between a bump in $\phi_{a}$ and a bump in $\phi_{b}(a b)$ 
is determined by the coupling term in the free energy. In the asymmetric case, the different values of $q$ mean that the size of the bumps are different in $\phi_{a}$ and $\phi_{b}$; hence, all possible interactions $a a, b b$, and $a b$ are different.

We begin by considering how the two order parameter profiles change as we alter their average values $\bar{\phi}_{a}=\bar{\phi}_{b}=\bar{\phi}$. Thus, we keep the concentration of the mixture fixed at $c=0.5$, where

$$
c=\frac{\bar{\phi}_{a}}{\bar{\phi}_{a}+\bar{\phi}_{b}} .
$$

We set the other parameter values to $\alpha=1, r=-0.9$, and $\eta=4$. In Fig. 11 we display typical results. We plot the normalized difference between the two order parameters $\Delta \hat{\phi}$ [as defined in Eq. (32)]. In the left column we show profiles from the symmetric case and in the middle column we display the profiles from the asymmetric system. In the right column we present the radial distribution functions, which are obtained by averaging over at least 50 runs, each with different realizations of the initial noise. The solid lines show the radial distribution functions for the symmetric case and the dashed lines show the asymmetric case. It is very apparent that this region of the parameter space shares many similarities with the one-component model in both $1 \mathrm{D}$ and 2D. If we select a small value of $\bar{\phi}$ we find localized peaks surrounded by vacant areas, as shown in Figs. 11(a) and 11(b). We observe a tendency for bumps in $\phi_{a}$ and in $\phi_{b}$ to sit pairwise next to each other resembling configurations occurring in mixtures of oppositely charged colloidal particles [34-36]. When $\bar{\phi}_{a} \approx \bar{\phi}_{b}$, the arrangement of the bumps also resembles snapshots of monovalent salts. It is very difficult to differentiate between the structures formed by the symmetric and asymmetric models for small values of $\bar{\phi}$. This is because, structurally, there is very little difference between the two cases. If we examine the radial distribution functions $g_{i j}$ (where $i, j=a, b$ ) for the symmetric and asymmetric systems [Fig. 11(c)] we observe that the average distance between the different bumps seems to be independent of the $q$ values (any difference between the curves is of the same order of magnitude as the statistical error). This is due to the large vacant areas, which means that there are not many bumps which are close to one another, especially between bumps of the same species ( $a a$ and $b b$ ).

As we increase the values of $\bar{\phi}$ we find that the number of bumps of both species increases. In Fig. 11(d)-11(f) we show the case where $\bar{\phi}=0.04$ and in Fig. $11(\mathrm{~g})-11(\mathrm{i})$ we show the case where $\bar{\phi}=0.06$. There is now a clear difference between the symmetric [Figs. 11(d) and $11(\mathrm{~g})$ ] and the asymmetric [Figs. 11(e) and 11(h)] cases. We observe a larger number of bumps in $\phi_{b}$ when $q_{b}=1.1$. This is because the larger value of $q$ reduces the length scale of the modulations, meaning that more bumps can be created before the value of $\phi_{b}$ becomes small (and negative) in vacant areas. There is an optimum value of $\phi_{a}$ and $\phi_{b}$ in the vacant (uniform) areas which depends on the parameter values. This explains why increasing the value of $\bar{\phi}$ increases the number of bumps (i.e., more modulations are needed in order to reach the optimum value of $\phi$ in the vacant regions). These intermediate values of $\bar{\phi}$ produce profiles with bump configurations that resemble real fluid structures. However, in stark contrast to the one-component system [shown in Fig. 7(b)], we now find the formation of chains of alternating bumps reminiscent of structures observed in charged fluids. The radial distribution functions in Figs. 11(f) and 11(i) show that the asymmetry induced by the different values of $q$ begins to take effect at these intermediate values of $\bar{\phi}$. We observe that statistically the bumps sit closer together in the asymmetric case, especially when two bumps in $\phi_{b}$ are next to each other ( $b b$, shown by green dashed line). This is due to the decreased size of the bumps in $\phi_{b}$, allowing them to sit slightly closer to their neighbors.

Increasing the average order parameter values $\bar{\phi}$ further, we begin to observe the formation of crystalline structures as shown by Figs. 11(j)-11(1). The interesting thing is that now we observe square ordering of the particles instead of the hexagonal ordering which is present in the regular PFC model and the one-component VPFC model. This implies that as we increase the concentration of one of the species from $c=0$ (almost a pure one-component system) to $c=0.5$ there must be a transition from hexagonal to square ordering of bumps; this is something we return to below in Sec. IIIC. Just as for the one-component system, we find that there are more modulations in $\phi_{b}$ when $q_{b}=1.1$. The profiles obtained with these parameter values resemble a compound crystal structure with vacancies and grain boundaries. The radial distribution functions in Fig. 11(1) show that the smaller size difference of the $\phi_{b}$ bumps in the asymmetric mixture has a large impact on the average position of the bumps in the structure compared to the symmetric mixture. This is because the higher concentration of particles forces them all closer together, resulting in all pairs of bumps $a a, b b$, and $a b$ being closer together.

In Figs. 12(a) and 12(b) we show the shape of the individual bumps in $\phi_{a}$ and $\phi_{b}$ obtained in the low density limit $\bar{\phi} \rightarrow 0$. To determine these radially symmetric profiles we fit functions of the form $\theta(x)$ as defined above in Eq. (21). The bumps in $\phi_{a}$ are virtually identical for both the symmetric and the asymmetric systems. The $\phi_{a}$ bump in the symmetric system and the $\phi_{b}$ bump in the asymmetric system decay to different values due to the different values of $\phi_{a}$ and $\phi_{b}$ in the vacant areas of the asymmetric system. We observe that in this two-component model, a bump in one order parameter profile coincides with a small depression in the other order parameter profile. This is caused by the coupling term, which means that the combination of a bump in one order parameter and a hole in the other order parameter reduces the free energy of the system. In Fig. 12(c) and 12(d) we show the shape of the "holes" which form in one profile under the bumps in the other order parameter field. These are determined the same way as the bump profiles: by fitting a function of the form shown in Eq. (21) to data points obtained from simulations. The depth of the holes is much smaller than the height of the bumps. This is because the vacancy term prevents large negative values of $\phi_{a}$ or $\phi_{b}$.

Using the fitted functions shown in Figs. 12(a)-12(d) we calculate effective pair potentials $V_{i j}(x)$ between the different particles in the system $(i, j=a, b)$. We do this by determining the free energy for a system containing two bumps and their corresponding holes at various distances apart. In Fig. 12(e) we display the effective pair potentials for both the symmetric (solid lines) and the asymmetric (dashed lines) systems. The results show that there is an attraction between all of the bumps, just as we found for the one-component system [Fig. 8(b)]. In 


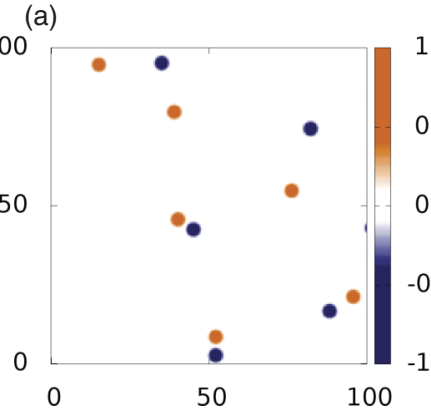

(d)

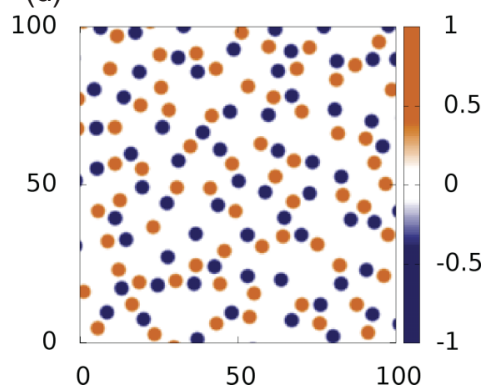

(g)

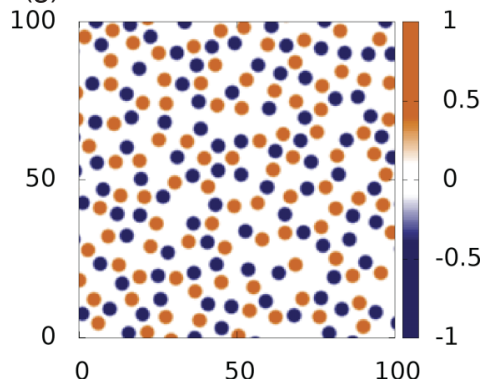

0

50

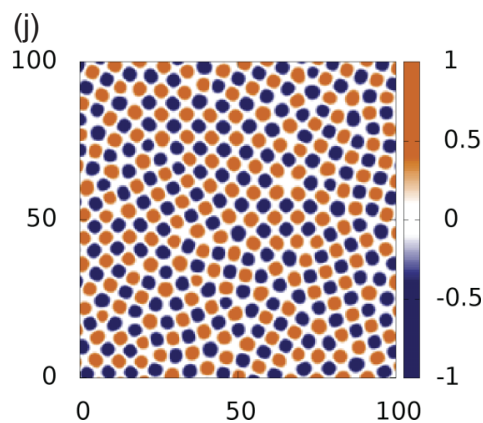

(b)

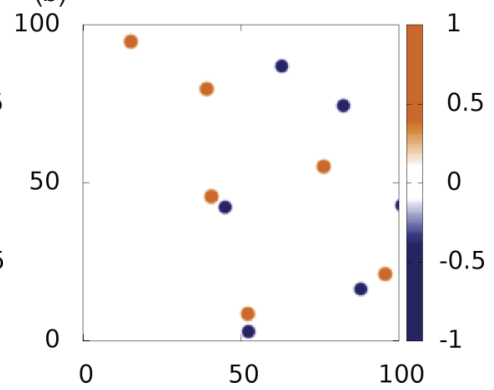

(c)

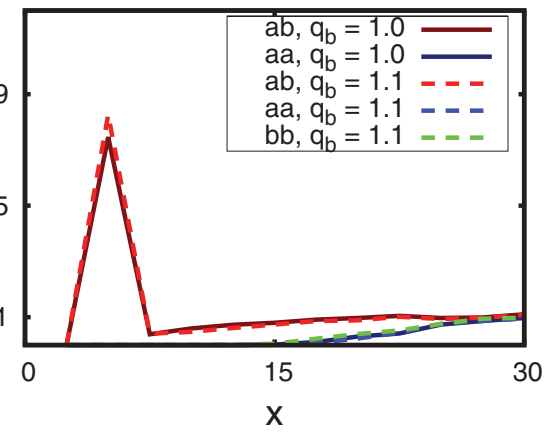

(e)

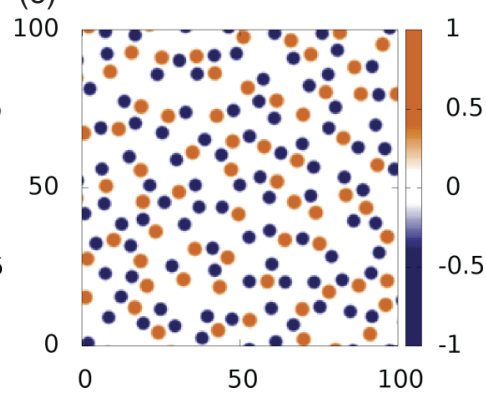

(h)
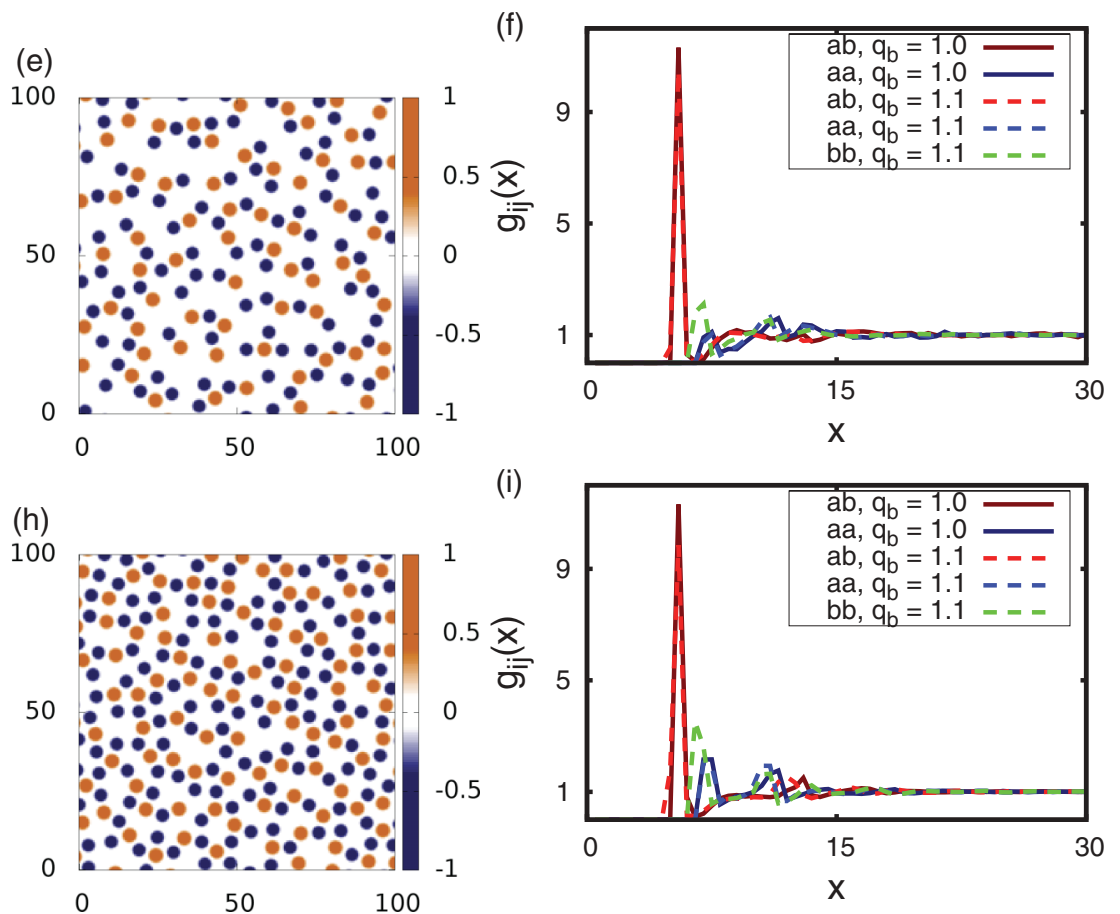

$(\mathrm{k})$
100

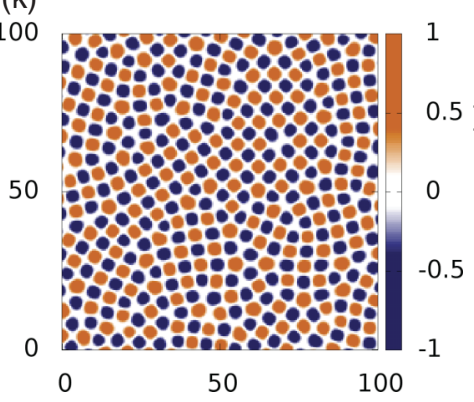

(I)

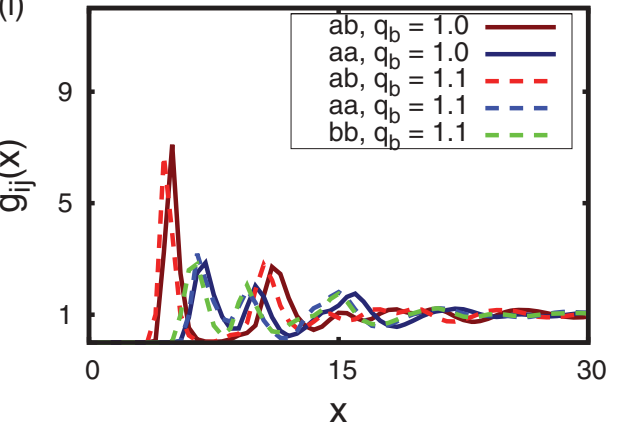

FIG. 11. (Color online) In the left-hand column we display typical simulation results for $\Delta \hat{\phi}$ for the symmetric case where $q_{a}=q_{b}=1$. In the middle column we display results from the asymmetric case where $q_{a}=1$ and $q_{b}=1.1$. The orange (light gray) regions show where there is a $\phi_{a}$ bump, while the blue (dark gray) show the $\phi_{b}$ bumps which are slightly smaller in the asymmetric mixture. In the right-hand column the radial distribution functions $g_{i j}(x)$ are shown for the symmetric case (solid lines) and the asymmetric case (dashed lines). The parameter values are $\alpha=1, \eta=4, r=-0.9, \bar{\phi}_{a}=\bar{\phi}_{b}=\bar{\phi}$, where (a)-(c) $\bar{\phi}=0$, (d)-(f) $\bar{\phi}=0.04$, (g)-(i) $\bar{\phi}=0.06$, and (j)-(l) $\bar{\phi}=0.15$.

both the symmetric and asymmetric cases we find that the attraction between two bumps from different species $(a b)$ is stronger and occurs at a smaller value of $x$ than that of two bumps of the same species $(a a$ and $b b)$. This explains the tendency for the bumps to form chains at intermediate values of $\phi_{a}$ and $\phi_{b}$ [Figs. 11(d), 11(e), 11(g), and 11(h)] and square ordered crystalline structures at larger values of $\phi_{a}$ and $\phi_{b}$ [Figs. 11(j) and 11(k)]. This is also consistent with the appearance of the large peak in $g_{a b}(x)$, which occurs at a smaller $x$ value than the main peaks in $g_{a a}(x)$ and $g_{b b}(x)$ [see Figs. 11(c), 11(f), 11(i), and 11(1)]. The effective pair potential $V_{a a}(x)$ is almost identical in the symmetric and the asymmetric 

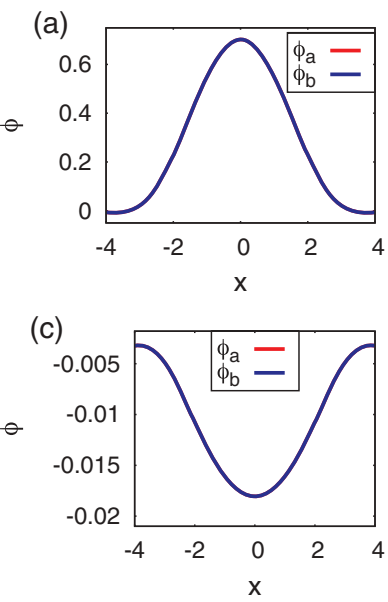

(b)
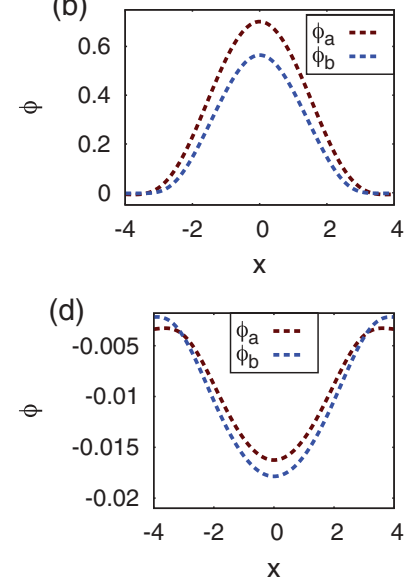

(e)

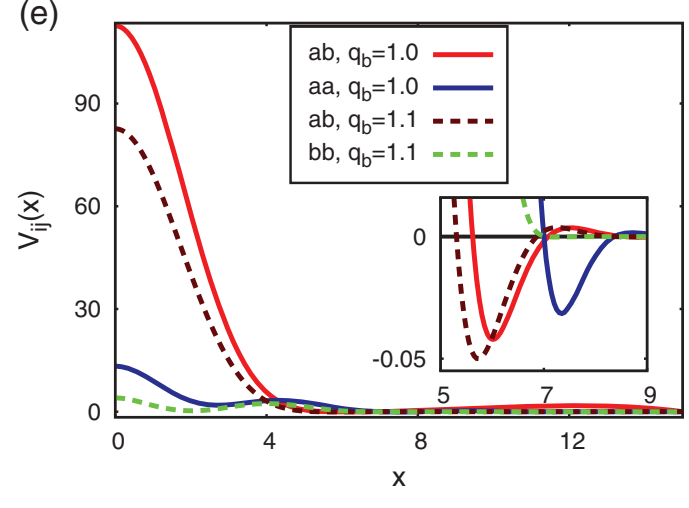

FIG. 12. (Color online) Fits to the shape of individual bumps (cf. Fig. 8) in the (a) symmetric, when $q_{b}=1$, and (b) asymmetric, when $q_{b}=1.1$, systems and the corresponding dips in the other order parameter profile which occur under the bumps in (c) the symmetric and (d) the asymmetric systems. In the symmetric cases (a) and (c) the $\phi_{a}$ and $\phi_{b}$ curves are identical everywhere. The bump profile in $\phi_{a}$ is virtually identical in the cases where $q_{b}=1$ and $q_{b}=1.1$. These fits are then used to calculate the effective pair potential between bumps, which are displayed in (e). The inset displays a magnification of the tails of $V_{i j}(x)$. The resulting pair potential $V(x)$ between two bumps in $\phi_{a}$ when $q_{b}=1.1$ lies on top of the $a a, q_{b}=1$ curve. The parameter values are $\alpha=1, r=-0.9, \eta=4$, and $\bar{\phi}_{a}=\bar{\phi}_{b}=0$.

systems. This suggests that the small hole which appears in $\phi_{b}$ has little effect on the interaction between the bumps. The major difference between the symmetric and asymmetric systems is that in the asymmetric mixture the minima of the pair potentials $V_{a b}(x)$ and $V_{b b}(x)$ are at smaller values of $x$ than in the symmetric mixture. This is due to the reduced size of the $\phi_{b}$ bumps in the latter. The minimum in $V_{b b}(x)$ is at a slightly larger value of $x$ than the minimum in $V_{a a}(x)$ and the attraction is also much weaker (in fact, it is so much weaker that the minimum is barely visible in this plot). This to some extent explains why the effect of the asymmetry is not visible for smaller values of $\phi_{a}$ and $\phi_{b}$, but becomes apparent for larger values of $\phi_{a}$ and $\phi_{b}$, where the vacant areas become smaller and we observe a close packing of the particles.

\section{Bond angles and the transition between hexagonal and square ordering}

In the 2D one-component model [Eqs. (1) and (4)] we observe hexagonally ordered structures for certain parameter values [Figs. 6(a) and 7(c)]. However, in the two-component model when $\bar{\phi}_{a}=\bar{\phi}_{b}$, we instead observe a square ordered crystalline structure which alternates between species $a$ and species $b$ [Figs. 11(j) and 11(k)]. Thus, as the composition of the mixture is varied we should see a transition or crossover from hexagonal to square ordering. The number of bumps observed in each field $\phi_{i}$ depends on the respective average value $\bar{\phi}_{i}$. When the concentration $c \approx 0$ or $c \approx 1$, where $c$ is defined in Eq. (33), (i.e., when either $\bar{\phi}_{b} \gg \bar{\phi}_{a}$ or $\bar{\phi}_{a} \gg \bar{\phi}_{b}$ ), then the resulting order parameter profile $\Delta \hat{\phi}(x)$ has many more bumps of one type than of the other, and in these two limits we again observe hexagonal ordering. Note that $c$ in Eq. (33) is not a bump concentration, but instead is a ratio between the two average order parameter values. As the $\phi_{i}$ may take a negative value, for $c=0$ there are still a few bumps of $a$ and similarly there are still some species $b$ bumps when $c=1$. When $c=0.5$ the number of bumps is roughly the same in both species for the symmetric case $\left(q_{a}=q_{b}\right)$, but this is not necessarily true for the asymmetric system $\left(q_{a} \neq q_{b}\right)$. When $\bar{\phi}_{a}=\bar{\phi}_{b}$ and $q_{a}<q_{b}$ there are more $b$ bumps than $a$ bumps.

In Figs. 13(a)-13(c) we show the order parameter $\Delta \hat{\phi}$ for varying values of $c$ for the symmetric mixture $\left(q_{a}=q_{b}\right)$. We fix the total "density" $\bar{\phi}_{a}+\bar{\phi}_{b}=0.24$ and investigate how the crystalline structures change as the concentration $c$ is varied. In Fig. 13(a), when $c=0$ we observe a profile which is dominated by species $b$ bumps. The crystal is hexagonally ordered with some defects (these tend to occur in the vicinity of the $\phi_{a}$ bumps). There are only a few $\phi_{a}$ bumps, which means the bumps in $b$ are usually sitting next to each other, resulting in them ordering themselves in a similar manner to that observed in the one-component model [Fig. 7(c)]. Increasing the value of $c$ from 0 to 0.25 , we observe a loss of crystalline structure, as shown in Fig. 13(b). The loss of long range order is clearly visible in the associated radial distribution functions (not shown). The profile in Fig. 13(b) shows a somewhat amorphous structure which appears to include both square and hexagonal ordering in equal measure. Increasing the concentration further to $c=0.5$, we observe a similar square ordering of bumps as in Figs. 11(j) and 11(k) [in Fig. 11(j), $q_{b}=1$, whereas in Fig. $11(\mathrm{k}), q_{b}=1.1$; all other parameter values are the same]. The crystalline structure in Fig. 13(c) at $\bar{\phi}_{a}=\bar{\phi}_{b}=0.12$ contains more vacancies and defects than the one in Fig. 11(j) at $\bar{\phi}_{a}=\bar{\phi}_{b}=0.15$, as both average order parameter values are smaller. Owing to the symmetry induced by choosing $q_{a}=q_{b}$ (i.e., $\phi_{a} \rightarrow \phi_{b}$ as $c \rightarrow 1-c$ ), a case with concentration $c$ is equivalent to the case with concentration $1-c$. Thus, Fig. 13(b) also shows the case $c=0.75$ if one interchanges the orange (light gray) and blue (dark gray) bumps. For this reason values $c>0.5$ are not shown.

For the asymmetric system the $c \rightarrow 1-c$ symmetry does not exist and we therefore show five cases for $c$ varying from 0 to 1 in Figs. 13(d) $c=0,13(\mathrm{e}) c=0.25,13(\mathrm{f}) c=0.5,13(\mathrm{~g})$ $c=0.75$, and $13(\mathrm{~h}) c=1$. We again observe a transition 


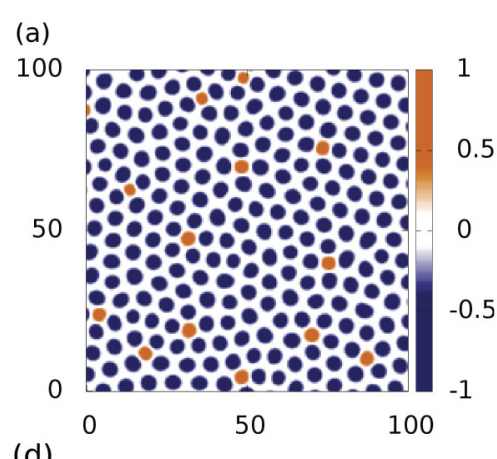

(b)

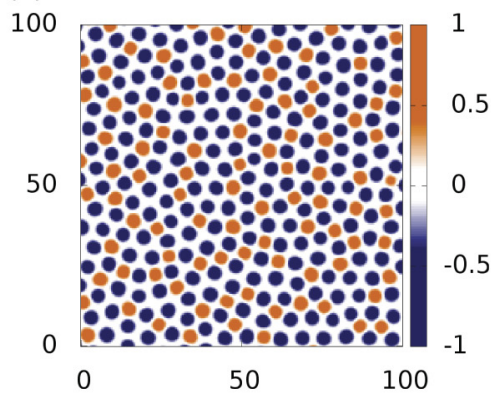

(e)
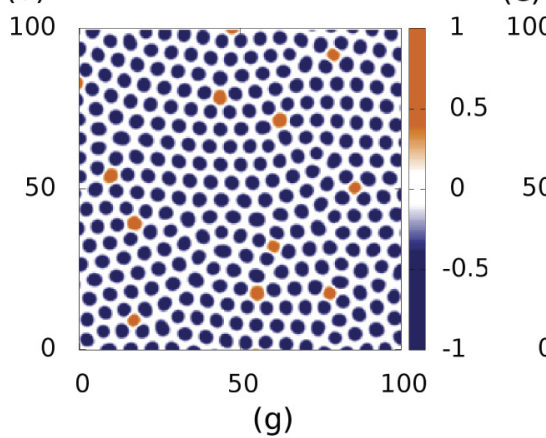

(g)

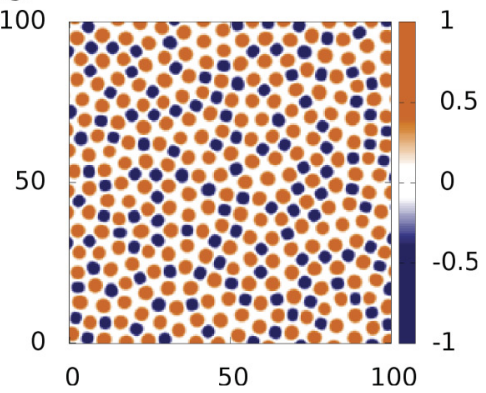

0

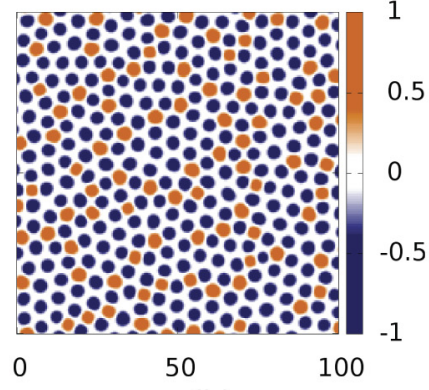

(h) (c)

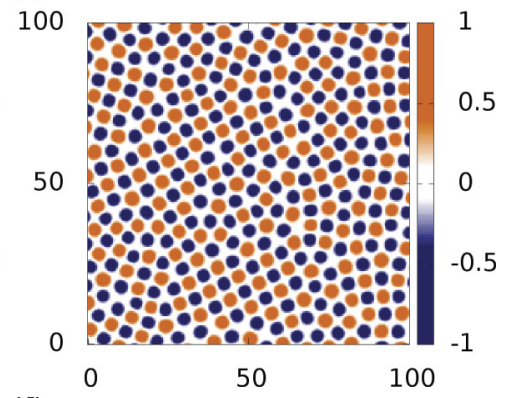

(f)

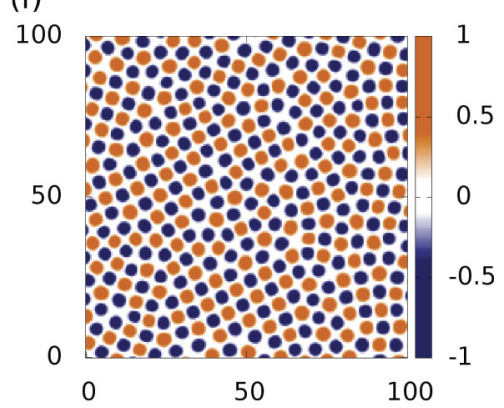

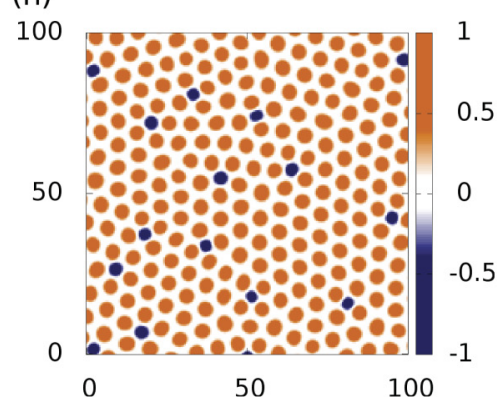

FIG. 13. (Color online) Plots of the order parameter $\Delta \hat{\phi}$, in which bumps in species $a$ appear in orange (light gray) and bumps in species $b$ appear in blue (dark gray), for a constant total order parameter value $\bar{\phi}_{a}+\bar{\phi}_{b}=0.24$. In (a)-(c) we show results from the symmetric mixture $\left(q_{a}=q_{b}=1\right)$, where the concentration of species $a$ is (a) $c=0$, (b) $c=0.25$, and (c) $c=0.5$. In (d)-(h) we show the results from the asymmetric mixture $\left(q_{a}=1\right.$ and $q_{b}=1.1$ ), where (d) $c=0$, (e) $c=0.25$, (f) $c=0.5,(\mathrm{~g}) c=0.75$, and (h) $c=1$. The parameter values are $\eta=4$ and $r=-0.9$.

from hexagonal ordering in Fig. 13(d) to square ordering in Fig. 13(f) and back to hexagonal ordering in Fig. 13(h) as the value of $c$ is increased from 0 to 1 . In between the highly structured states we observe the mixed ordered states [Figs. 13(e) and 13(g)] that were also present in the symmetric system. By eye, it is very difficult to pick out the differences between the symmetric and the asymmetric cases. As previously discussed, the different value of $q_{b}$ in the asymmetric system changes the shape, size, and quantity of $b$ bumps. In order to characterize and better understand the organization of the crystalline structures that are formed, we require a measure which may be used to quantify the structures and distinguish between hexagonal and square ordering in both the symmetric and the asymmetric systems. To do this, we use Delaunay triangulation $[37,38]$ to calculate the distribution of the bond angles $p(\Theta)$ between nearest neighbors. We could have used other measures from stochastic geometry [39], which were used to characterize the hexagon-square transition in Bénard convection [40].

The Delaunay triangulation is a triangulation of points (in our case the coordinates of the peaks of the bumps in both order parameter fields), which maximizes the minimum angles of every triangle (i.e., avoids "skinny" triangles). This triangulation can be calculated from the Voronoi diagram [37,38] of any set of points on a $2 \mathrm{D}$ plane. The Voronoi diagram is a set of polygons, where each polygon represents an area in $2 \mathrm{D}$ space which is closer to a particular point than to any of the other points (i.e., the locus of points contained in each polygon is closer to the bump inside the polygon than any other bump). In Fig. 14 we show an example of how we calculate the Delaunay triangulation for a given order parameter profile. The example shows the triangulation for a one-component profile (as the pairing between bumps in the two-component model makes the triangulation harder to see) but the process is applied in the same manner to the two-component model. We take the coordinates of all the bumps to be our points on a $2 \mathrm{D}$ plane. We then calculate the Voronoi diagram [shown as the light blue (light gray) lines in Fig. 14(b)], which can be used to calculate the Delaunay triangulation [shown as the red (dark gray) lines in Fig. 14(b)]. This can be done using any of the algorithms outlined in Refs. [37,38]. For an efficient method of calculating Voronoi diagrams and Delaunay triangulations, see Ref. [41]. 
(a)

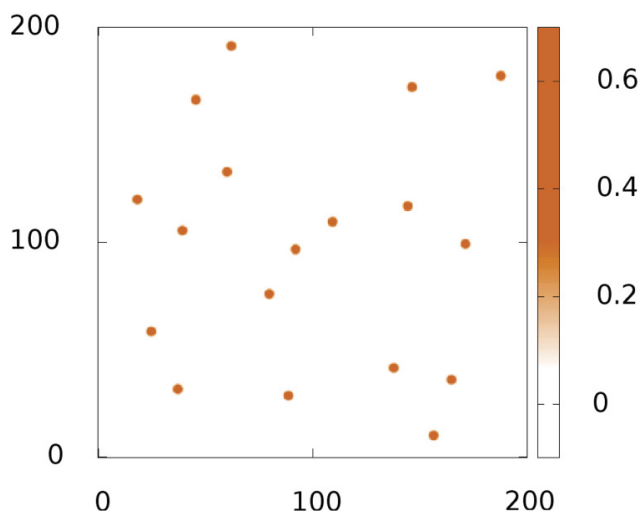

(b)

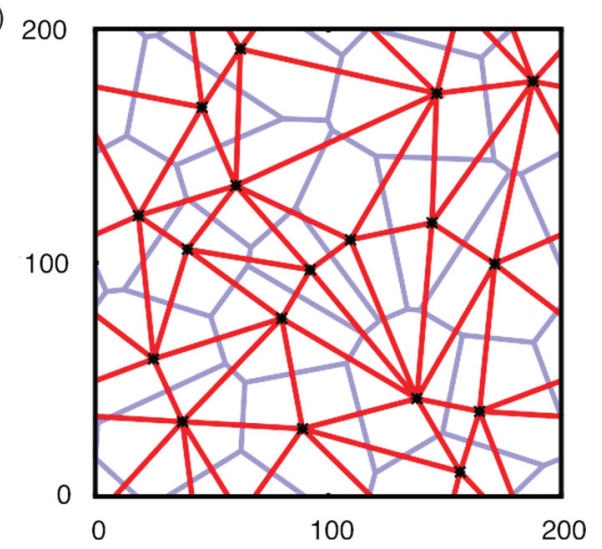

FIG. 14. (Color online) An example Delaunay triangulation is shown for a simple one-component case. In (a) we display a typical order parameter profile for the one-component model where we observe isolated peaks. The coordinates of the maxima are calculated; these are shown as black points in (b). In (b) we show the Voronoi diagram as a light blue (light gray) polygon network and the Delaunay triangulation as red (dark gray) triangles for this particular set of coordinates.

(Note that Delaunay triangulation becomes degenerate when points appear in certain lines of symmetry. However, the initial noise added to the order parameter fields prevents bumps from forming in perfect symmetry.) We use the statistics of the triangles in the Delaunay triangulation to characterize the structures produced by the bumps.

We extract three quantities from the triangulation: the area of the triangles, the length of the sides, and the angles in each of the triangles. This information is gathered for five different realizations of the initial noise profile for systems of size $200 \times 200$ and the information is sorted into bins. From these bins we obtain the probability distribution function for each quantity. Comparing the different distributions for various values of $c$ allows us to observe how the triangles in the triangulation change as we go from hexagonal to square ordering. Here we concentrate on the probability distributions of the angles in the triangulation to characterize the crystalline structures. For results from the other measures, see Ref. [42].

In Fig. 15 we display the probability distribution $p(\Theta)$ for the triangle corner angles, as the concentration $c$ is varied from 0 to 1 . We show results for the symmetric (solid red line) and the asymmetric (dashed blue line) systems. The distribution of the angles of the triangles clearly shows the transition between hexagonal and square ordering. When $c=0$ we observe hexagonal ordering in both the symmetric and the asymmetric systems, which results in the formation of roughly equilateral triangles in the Delaunay triangulation. This produces angle distributions which have a single peak at $60^{\circ}$, as shown in Fig. 15(a). As the value of $c$ increases the structure changes to square ordering, transforming the triangles into right-angled triangles. Hence, the angle distribution changes and we observe a peak slightly above the value $45^{\circ}$ and another peak (half the size) slightly below $90^{\circ}$, as shown in Fig. 15(c). Increasing the concentration further to $c=1$ restores the hexagonal ordering; hence, the angle distribution returns to the single peak at $60^{\circ}$ [Fig. 15(e)]. In between the purely hexagonal and the purely square ordered structures we observe states where the distribution of bond angles is more evenly spread, with small peaks occurring just above $45^{\circ}$, at around $60^{\circ}$ and just below $90^{\circ}$ [Figs. 15(b) and 15(d)]. These represent the somewhat amorphous structures which lack the long range ordering which is present in the hexagonally and square ordered structures. The position of these peaks in the bond angle distributions $p(\Theta)$ does not depend on the quantity or size of the bumps and so the peaks occur in (almost) the same position for the symmetric and the asymmetric systems for all values of $c$ (this is not the case for the area or length distributions). This makes the bond angle distributions ideal for comparing the structure of bump formations in different systems. On comparing the symmetric and the asymmetric cases we observe that $p(\Theta)$ appears smoother in the symmetric case. The distribution function $p(\Theta)$ has a more jagged appearance for the asymmetric mixture, which we believe is due to the fact that there are different sized bumps in this mixture, making it more difficult for the bumps to organize themselves into regular structures. In Figs. 15(a) and 15(e) the distributions appear very similar for the symmetric and the asymmetric cases; however, in the other distributions [in particular, Figs. 15(b) and 15(d)] we observe a distinct difference in the height of the three peaks. This suggests that the transition between the different ordered states occurs differently in the symmetric and the asymmetric systems.

To examine more closely the transition from the hexagonal to the square ordered states we introduce an order parameter $\Phi$ which is calculated from the distribution of the angles from the Delaunay triangulation. We integrate the angle distributions over three regions which cover the three different peaks (these regions are determined arbitrarily from close examination of the angle distributions in Fig. 15) and define the quantities

$$
\begin{aligned}
& R_{0}=\int_{25}^{53} p(\Theta) d \Theta, \quad R_{1}=\int_{53}^{72} p(\Theta) d \Theta \\
& R_{2}=\int_{72}^{115} p(\Theta) d \Theta .
\end{aligned}
$$

We then define the order parameter $\Phi$ in the following way:

$$
\Phi=\frac{R_{0}+R_{2}}{R_{1}} .
$$




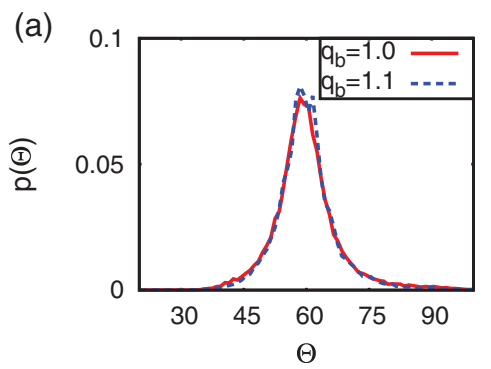

(d) (b)

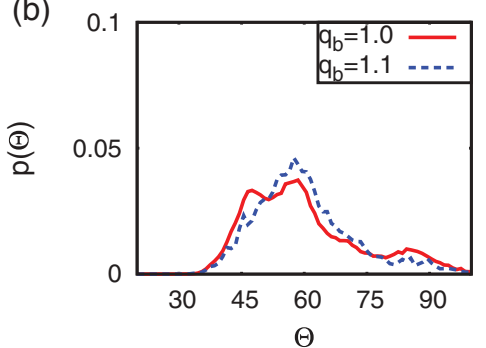

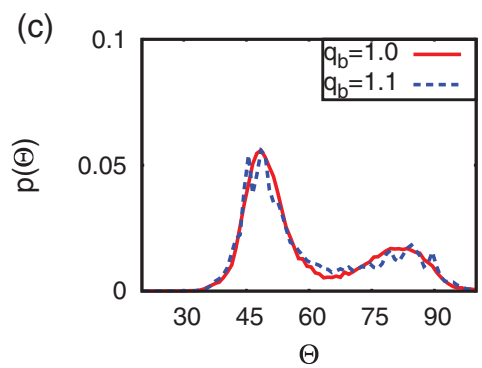
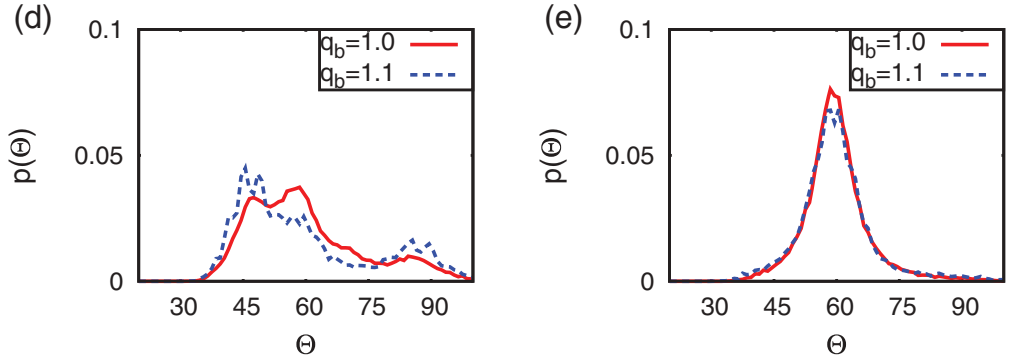

FIG. 15. (Color online) The bond angle distribution $p(\Theta)$ for a constant total order parameter value $\bar{\phi}_{a}+\bar{\phi}_{b}=0.24$. The concentration of species $a$ is (a) $c=0$, (b) $c=0.25$, (c) $c=0.5$, (d) $c=0.75$, and (e) $c=1$ (corresponding to the simulation snapshots shown in Fig. 13). Results for the symmetric system are shown as red solid lines and the asymmetric system results are shown as blue dashed lines. The parameter values are $q_{a}=1, \eta=4$, and $r=-0.9$.

When a structure consists of mainly hexagonal configurations of bumps the value of $\Phi$ is small (since $\Phi \rightarrow 0$ as $R_{0} \rightarrow 0$ and $R_{2} \rightarrow 0$ ) and when a profile is dominated by square ordering the value of $\Phi$ is large (since $\Phi \rightarrow \infty$ as $R_{1} \rightarrow 0$ ). Calculating this quantity for the angle distributions for different values of $c$ gives us a measure for the hexagonal vs square ordering of the bumps.

In Fig. 16 we show how the order parameter $\Phi$ changes with the concentration $c$ for the symmetric (solid red line) and the asymmetric (dashed blue line) mixtures. Both curves show a smooth continuous transition from hexagonal ordering to square ordering and back again. The different sized bumps in the asymmetric system break the symmetry around $c=0.5$ and we observe that the maximum (which corresponds to the strongest square ordering) occurs at around $c \approx 0.6$ and

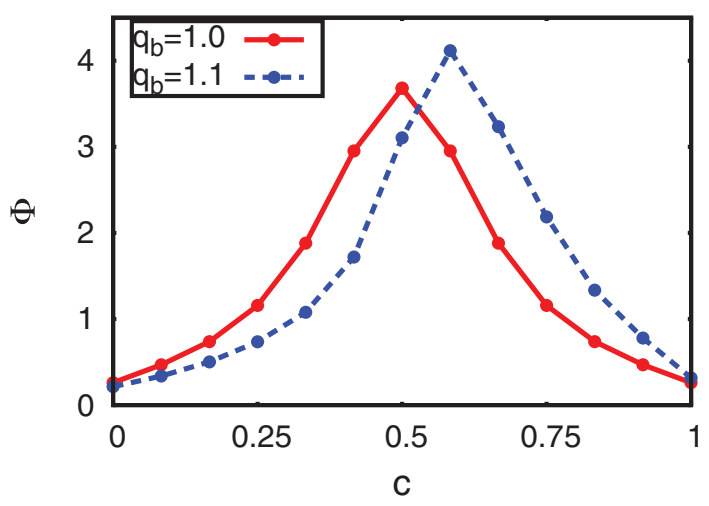

FIG. 16. (Color online) Plot showing the order parameter $\Phi$ [defined in Eq. (35)] as a function of the concentration $c$ of species $a$, where $\bar{\phi}_{a}+\bar{\phi}_{b}=0.24$. The red solid line and points show the symmetric case and the blue dashed line and points show the asymmetric case. The parameter values are $q_{a}=1, \eta=4$, and $r=-0.9$. is actually higher than the peak in the symmetric case. The transition to and from square ordering appears to be slightly sharper in the asymmetric case. Even though there is a difference in the transition between the different ordered states in the symmetric and the asymmetric mixtures, they appear to be qualitatively similar. It may be the case that for a larger difference in the values of $q_{a}$ and $q_{b}$ a different type of transition from hexagonal to square ordering might occur, for example, a discontinuous transition. However, the effect of varying the ratio $q_{a} / q_{b}$ is not studied in detail here.

\section{CONCLUSIONS}

In this paper we have investigated the VPFC model and its application to materials modeling. We first considered the one-component model proposed by Chan et al. in Ref. [13]. We determined the linear stability of the homogeneous state and discussed the dispersion relation. We examined the phase behavior in 1D and calculated the phase diagram, computing exactly the location of the tricritical point between the homogeneous and periodic states, and identified the region of phase space where localized structures occur. Focusing on the latter region of the phase diagram, we investigated the localized steady state profiles and discussed the slanted homoclinic snaking which occurs in the bifurcation diagrams. The one-component model was also studied in 2D and we determined the phase diagram, radial distribution functions, and effective pair potentials from our simulation data. Some of the behavior we have identified-the presence of transitions resembling transitions from a solid phase to a liquid phase and then to a gaslike phase-replicates behavior observed in nonconserved systems [43]. In Sec. III of the paper, we extended the model to include two coupled order parameter fields. We have considered how the coupling affects the linear stability of flat profiles and then briefly touched on 
the phase behavior of this two-component model. We have focused on the bump structures which form, considering both a symmetric mixture where the bumps are of equal size and an asymmetric system where one of the bump species is slightly smaller than the other species. The radial distribution functions and effective pair potentials for these systems are somewhat similar to those in binary mixtures of oppositely charged colloidal particles. We have investigated how varying the concentration $c$ of the mixture produces a crossover from hexagonal to square ordered crystalline structures and how the transition differs between the symmetric and the asymmetric systems.

A key issue on which we should comment concerns the question of what precisely does the order parameter profile $\phi(\mathbf{x}, t)$ in the VPFC model represent? In the regular PFC model, the phase with the uniform flat profile is taken to represent the liquid phase, while the bump phase corresponds to the crystalline solid. This interpretation is underpinned by the fact that the regular PFC can be derived from density functional theory (DFT) [2] and dynamical density functional theory (DDFT) [10], which is a theory for the dynamics of a system of interacting Brownian (colloidal) particles [44-47]. DFT [48-50] is a statistical mechanics theory for the one-body number density $\rho(\mathbf{x})$ of a system of particles, where $\rho(\mathbf{x})=$ $\langle\hat{\rho}(\mathbf{x})\rangle$ and where $\hat{\rho}(\mathbf{x})=\sum_{i} \delta\left(\mathbf{x}-\mathbf{x}_{i}\right)$ is the density operator and $\langle\cdot\rangle$ denotes a statistical ensemble average [48]. The central quantity in DFT is the Helmholtz free energy functional $F[\rho]$ and the equilibrium fluid density profile $\rho(\mathbf{x})$ is that which minimizes the grand free energy $\Omega[\rho]=F[\rho]-\mu \int \mathrm{d} \mathbf{x} \rho(\mathbf{x})$. The DDFT for Brownian particles [44-47] takes as input this functional and so yields the correct equilibrium fluid density profile. Making a truncated gradient expansion approximation for $F[\rho]$, expanding the free energy around that of a reference liquid state with uniform density $\rho_{0}$, one can argue that the free energy is approximately given by Eqs. (2) and (3), where the order parameter $\phi(\mathbf{x}) \propto \rho(\mathbf{x})-\rho_{0}$. Thus, it is clear that in a bulk liquid, where $\rho(\mathbf{x})$ is a constant, so too is $\phi(\mathbf{x})$ a constant and in the solid phase, where $\rho(\mathbf{x})$ consists of a periodic array of density peaks, then $\phi(\mathbf{x})$ also contains periodic modulations. However, there are some problems extending this interpretation to the VPFC. Consider, for example, Fig. 7(a), where we see a few isolated localized peaks surrounded by a uniform background where $\phi(\mathbf{x}) \approx 0$. Maintaining the above PFC interpretation, this would correspond to a few individual "frozen" particles, surrounded by a fluid of mobile particles. One might be tempted to think of this as some sort of glass transition [50-52], but the glass transition is a collective phenomenon: In a glass one does see "dynamical heterogeneity," that is, regions where the particles are totally jammed and other regions which are more mobile, but to our knowledge one never sees a single particle that is jammed on its own surrounded by more mobile particles. Thus, it may be possible to assume this interpretation may be maintained for the VPFC, that is, by considering the localized peaks surrounded by a uniform background to be a dynamically heterogeneous glassy system, but there are problems with this point of view.

An alternative interpretation for the order parameter profile in the VPFC model is that $\phi(\mathbf{x})$ is related to a coarse-grained density profile (rather than an ensemble average density profile $)$ for the system $\tilde{\rho}(\mathbf{x}, t)$, that is, $\phi(\mathbf{x}, t) \propto \tilde{\rho}(\mathbf{x}, t)$. Following Ref. [47], we may define the temporally coarse-grained density profile for a system of Brownian colloidal particles as $\tilde{\rho}(\mathbf{x}, t)=\int K\left(t-t^{\prime}\right) \hat{\rho}(\mathbf{x}, t) \mathrm{d} t^{\prime}$, where $K(t)$ is a normalized function of finite support which defines a time window over which the density is coarse grained. One can then argue [47] that the time evolution equations for $\tilde{\rho}(\mathbf{x}, t)$ must be very similar or even the same as the DDFT equations for the time evolution of the ensemble average density $\rho(\mathbf{x}, t)$, as long as the width in time $\tau$ of $K(t)$ is large enough. By choosing the time $\tau$ so that it is large compared to the time between the colloidal particles receiving Brownian "kicks" from the solvent, but is short compared to the diffusive time scale, corresponding to the typical time for a particle to diffuse a distance equal to its own diameter, then the coarse-grained density $\tilde{\rho}(\mathbf{x}, t)$ and the order parameter $\phi(\mathbf{x}, t)$ will be quantities which contain peaks, each of which correspond to an individual particle in the system [53]. Thus, in a low density colloidal suspension one should see isolated peaks in the coarse-grained density, surrounded by regions where $\phi(\mathbf{x}, t) \approx 0$, corresponding to no particles being present in that region of the system. This is the justification for the interpretation made by Chan et al. in Ref. [13], that the peaks in the order parameter correspond to particles and the uniform background corresponds to a portion of solvent free of particles. In order to observe the long time Brownian motion of the particles in this description, one should add a stochastic noise term to the dynamical equations for the system (23), which continuously drives the system (as opposed to the small amount of noise that is present in our initial order parameter profiles). However, in numerical simulations there can be problems with such an approach, because the particles can become pinned in place by the discrete grid on which they are defined, and so do not move. We did not make a detailed investigation of the VPFC model with additional noise. Further issues arise as the noise renormalizes the parameters of the continuum model.

There are state points in the PFC and VPFC phase diagram where all possible interpretations of $\phi$ break down: These are the state points where the equilibrium state is the stripe or the hole phase, such as those displayed in Fig. 10. Systems of spherical particles do not have an ensemble average density profile $\rho$ nor a coarse-grained density profile $\tilde{\rho}$ with stripes or holes, unless the particles in the system interact via pair potentials containing competing attractive and repulsive parts $[18,54,55]$. We must conclude that for the parameter values corresponding to these state points, the gradient expansion that is implicit in the PFC and VPFC free energy functionals has broken down and that these order parameter profiles are unphysical.

The radial distribution functions for the one-component model displayed in Fig. 7 (see also Fig. 5 of Ref. [13]) are very similar to those in real fluids. We observe static correlations which are very similar to what one observes in fluids. Increasing the value of $\bar{\phi}$ increases the number of bumps and close packing causes long range (crystalline) ordering of the bumps. Calculating the effective pair potential between isolated pairs of bumps, we find a pair potential having an attractive minimum at a pair separation distance which is slightly larger than the diameter of the bumps. Thus, the interactions and correlations between bumps share certain 
features with some colloidal fluids [56]. We also extend the model to consider a two-component mixture, with a simple repulsive coupling between the two order parameter profiles. At low values of $\bar{\phi}$ the bumps commonly appear in pairs and at intermediate values they tend to form chains. At higher values of $\bar{\phi}$ the system exhibits crystalline ordering. The appearance of these structures is somewhat reminiscent of the arrangement of the particles in a binary mixture of oppositely charged colloidal particles (see, e.g., Refs. [34,57] and references therein). The radial distribution functions and the effective pair potentials show that there is a fairly strong attraction between bumps of the opposite species $a$ and $b$. The minimum in the $a b$ effective pair potential is at a shorter pair separation distance than the minimum in the $a a$ and $b b$ pair potentials and so we observe square ordering when the concentration $c \approx 1 / 2$ and $\bar{\phi}$ is high enough for the bumps to pack into a crystalline structure. However, when $c \approx 0$ or $c \approx 1$, we observe hexagonal ordering and so we observe a transition from hexagonal to square ordering as the concentration $c$ is varied. We find that this transition occurs smoothly but can become skewed by changing the size of one of the species of bumps $\left(q_{a} \neq q_{b}\right)$.
It would be interesting to further investigate the effect that varying the ratio $q_{a} / q_{b}$ has on the structures which form. In particular, determining the range of values of $q_{a}$ and $q_{b}$ for which bump profiles form in the $2 \mathrm{D}$ system would allow one to determine the range of size ratios of particles (bumps) that can be modeled. The transition between hexagonal and square structures could then be studied for systems with very different sized bumps and if the VPFC in this regime continues to be able to model mixtures of charged colloidal particles, then a wide range of different crystal structures should be observed [34].

Note also that the localized structures that we observe are not a unique property of the VPFC model but are, in fact, also present in the regular PFC model for a small range of parameter values outside the limit of linear stability. This is something that we will focus on in future work.

\section{ACKNOWLEDGMENTS}

This work was supported by the EU via the ITN MULTIFLOW (PITN-GA-2008-214919). M.J.R. also gratefully acknowledges support from EPSRC and A.J.A. thanks RCUK for support.
[1] K. R. Elder, M. Katakowski, M. Haataja, and M. Grant, Phys. Rev. Lett. 88, 245701 (2002).

[2] K. R. Elder and M. Grant, Phys. Rev. E 70, 051605 (2004).

[3] G. Tegze et al., Soft Matter 7, 1789 (2011).

[4] J. Mellenthin, A. Karma, and M. Plapp, Phys. Rev. B 78, 184110 (2008).

[5] K.-A. Wu, M. Plapp, and P. W. Voorhees, J. Phys.: Condens. Matter 22, 364102 (2010).

[6] J. Swift and P. C. Hohenberg, Phys. Rev. A 15, 319 (1977).

[7] E. Knobloch, Phys. Rev. A 40, 1549 (1989).

[8] P. C. Matthews and S. M. Cox, Nonlinearity 13, 1293 (2000).

[9] K. R. Elder, N. Provatas, J. Berry, P. Stefanovic, and M. Grant, Phys. Rev. B 75, 064107 (2007).

[10] S. van Teeffelen, R. Backofen, A. Voigt, and H. Löwen, Phys. Rev. E 79, 051404 (2009).

[11] Z.-F. Huang, K. R. Elder, and N. Provatas, Phys. Rev. E 82, 021605 (2010).

[12] H. Ohnogi and Y. Shiwa, Physica D 237, 3046 (2008).

[13] P. Y. Chan, N. Goldenfeld, and J. Dantzig, Phys. Rev. E 79, 035701 (2009).

[14] J. Berry and M. Grant, Phys. Rev. Lett. 106, 175702 (2011).

[15] G. Stell, J. Stat. Phys. 78, 197 (1995).

[16] A. Ciach, W. T. Gózdz, and R. Evans, J. Chem. Phys. 118, 3702 (2003).

[17] A. J. Archer, C. N. Likos, and R. Evans, J. Phys.: Condens. Matter 16, L297 (2004).

[18] A. J. Archer, D. Pini, R. Evans, and L. Reatto, J. Chem. Phys. 126, 014104 (2007).

[19] J. Burke and E. Knobloch, Phys. Rev. E 73, 056211 (2006).

[20] J. Burke and E. Knobloch, Chaos 17, 037102 (2007).

[21] J. Burke and E. Knobloch, Phys. Lett. A 360, 681 (2007).

[22] E. Knobloch, Nonlinearity 21, T45 (2008).

[23] E. Doedel et al., Caltech, technical report, 2011 (unpublished).
[24] J. Burke and E. Knobloch, in Dynamical Systems, Differential Equations and Applications, Discrete and Continuous Dynamical Systems-Suppl. September, edited by X.-J. Hou et al. (AIMS, Springfield, 2009), pp. 109-117.

[25] J. H. P. Dawes, SIAM J. Appl. Dyn. Syst. 7, 186 (2008).

[26] D. Lo Jacono, A. Bergeon, and E. Knobloch, J. Fluid Mech. 687, 595 (2011).

[27] U. Thiele, A. J. Archer, M. J. Robbins, H. Gomez, and E. Knobloch (unpublished).

[28] M. P. Allen and D. J. Tildesley, Computer Simulation of Liquids (Clarendon Press, Oxford, 1999).

[29] G. I. Tóth et al., J. Phys.: Condens. Matter 22, 364101 (2010).

[30] L. Gránásy, G. Tegze, G. I. Tóth, and T. Pusztai, Philos. Mag. 91, 123 (2011).

[31] S. Muralidharan and M. Haataja, Phys. Rev. Lett. 105, 126101 (2010).

[32] A. Pototsky, M. Bestehorn, D. Merkt, and U. Thiele, J. Chem. Phys. 122, 224711 (2005).

[33] M. J. Robbins, A. J. Archer, and U. Thiele, J. Phys.: Condens. Matter 23, 415102 (2011).

[34] M. E. Leunissen et al., Nature (London) 437, 235 (2005).

[35] A. P. Hynninen, C. G. Christova, R. van Roij, A. van Blaaderen, and M. Dijkstra, Phys. Rev. Lett. 96, 138308 (2006).

[36] A. P. Hynninen, M. E. Leunissen, A. van Blaaderen, and M. Dijkstra, Phys. Rev. Lett. 96, 018303 (2006).

[37] M. de Berg, M. van Kreveld, M. Overmars, and O. Schwarzkopf, Computational Geometry: Algorithms and Applications (Springer, Berlin, 1997).

[38] Ø. Hjelle and M. Dæhlen, Triangulations and Applications (Springer, Berlin, 2006).

[39] D. Stoyan, W. S. Kendall, and J. Mecke, Stochastic Geometry and Its Applications, 2nd ed. (Wiley, West Sussex, 1995).

[40] U. Thiele and K. Eckert, Phys. Rev. E 58, 3458 (1998). 
[41] A. Bowyer, Comput. J. 24, 162 (1981).

[42] M. J. Robbins, Ph.D. thesis, Loughborough University, 2012.

[43] U. Bortolozzo, M. G. Clerc, and S. Residori, New J. Phys. 11, 093037 (2009).

[44] U. M. B. Marconi and P. Tarazona, J. Chem. Phys. 110, 8032 (1999).

[45] U. M. B. Marconi and P. Tarazona, J. Phys.: Condens. Matter 12, A413 (2000).

[46] A. J. Archer and R. Evans, J. Chem. Phys. 121, 4246 (2004).

[47] A. J. Archer and M. Rauscher, J. Phys. A 37, 9325 (2004).

[48] R. Evans, Adv. Phys. 28, 143 (1979).

[49] R. Evans, in Fundamentals of Inhomogeneous Fluids, edited by D. Henderson, Chap. 3 (Dekker, New York, 1992).

[50] J. P. Hansen and I. R. McDonald, Theory of Simple Liquids, 3rd ed. (Academic Press, London, 2006).

[51] L. Berthier and G. Biroli, Rev. Mod. Phys. 83, 587 (2011).
[52] A. Cavagna, Phys. Rep. 476, 51 (2009).

[53] Taking this point of view, one might then conclude that the parameter $q$ in the VPFC, which determines the size of the bumps, must be related to $\tau$, the width of the coarse-graining time window. However, since the VPFC is not derived by starting from the equations of motion for particles and then following consistently this coarse-graining approach, this interpretation cannot be maintained, because varying $q$ affects the whole thermodynamics of the model.

[54] A. J. Archer, Phys. Rev. E 78, 031402 (2008).

[55] A. J. Archer and N. B. Wilding, Phys. Rev. E 76, 031501 (2007).

[56] J.-L. Barrat and J. P. Hansen, Basic Concepts for Simple and Complex Liquids (Cambridge University Press, Cambridge, 2003).

[57] P. Bartlett and A. I. Campbell, Phys. Rev. Lett. 95, 128302 (2005). 